University of South Florida

DIGITAL COMMONS

Digital Commons @ University of

@ UNIVERSITY OF SOUTH FLORIDA

South Florida

$9-1-2009$

\title{
Evaluation of Electronic Data Recorder for Incident Investigation, Driver Performance, and Vehicle Maintenance
}

CUTR

Follow this and additional works at: https://digitalcommons.usf.edu/cutr_nctr

\section{Recommended Citation}

"Evaluation of Electronic Data Recorder for Incident Investigation, Driver Performance, and Vehicle Maintenance," National Center for Transit Research (NCTR) Report No. CUTR-NCTR-RR-2008-07, Center for Urban Transportation Research, University of South Florida, 2009.

DOI: https://doi.org/10.5038/CUTR-NCTR-RR-2008-07

Available at: https://scholarcommons.usf.edu/cutr_nctr/149

This Technical Report is brought to you for free and open access by the National Center for Transit Research (NCTR) Archive (2000-2020) at Digital Commons @ University of South Florida. It has been accepted for inclusion in Research Reports by an authorized administrator of Digital Commons @ University of South Florida. For more information, please contact digitalcommons@usf.edu. 


\section{EVALUATION OF ELECTRONIC DATA RECORDERS FOR INCIDENT INVESTIGATION, DRIVER PERFORMANCE, AND VEHICLE MAINTENANCE}

Project \#BD549-50

\section{FINAL REPORT}

Prepared for the

Florida Department of Transportation

Research Center

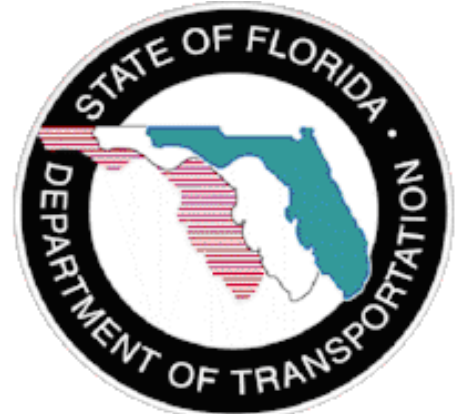

Project Manager:

Robert Westbrook

Prepared by the

National Center for Transit Research

Center for Urban Transportation Research

University of South Florida

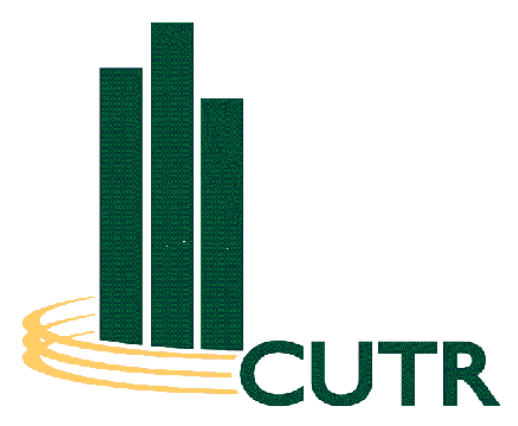

September 2009 


\section{$\underline{\text { Disclaimer }}$}

The opinions, findings, and conclusions expressed in this publication are those of the authors who are responsible for the facts and accuracy of the data presented herein. The contents do not necessarily reflect the views and policies of the Florida Department of Transportation or the

Research and Innovative Technology Administration. This report does not constitute a standard, specification, or regulation 
USF CENTER FOR URBAN TRANSPORTATION RESEARCH

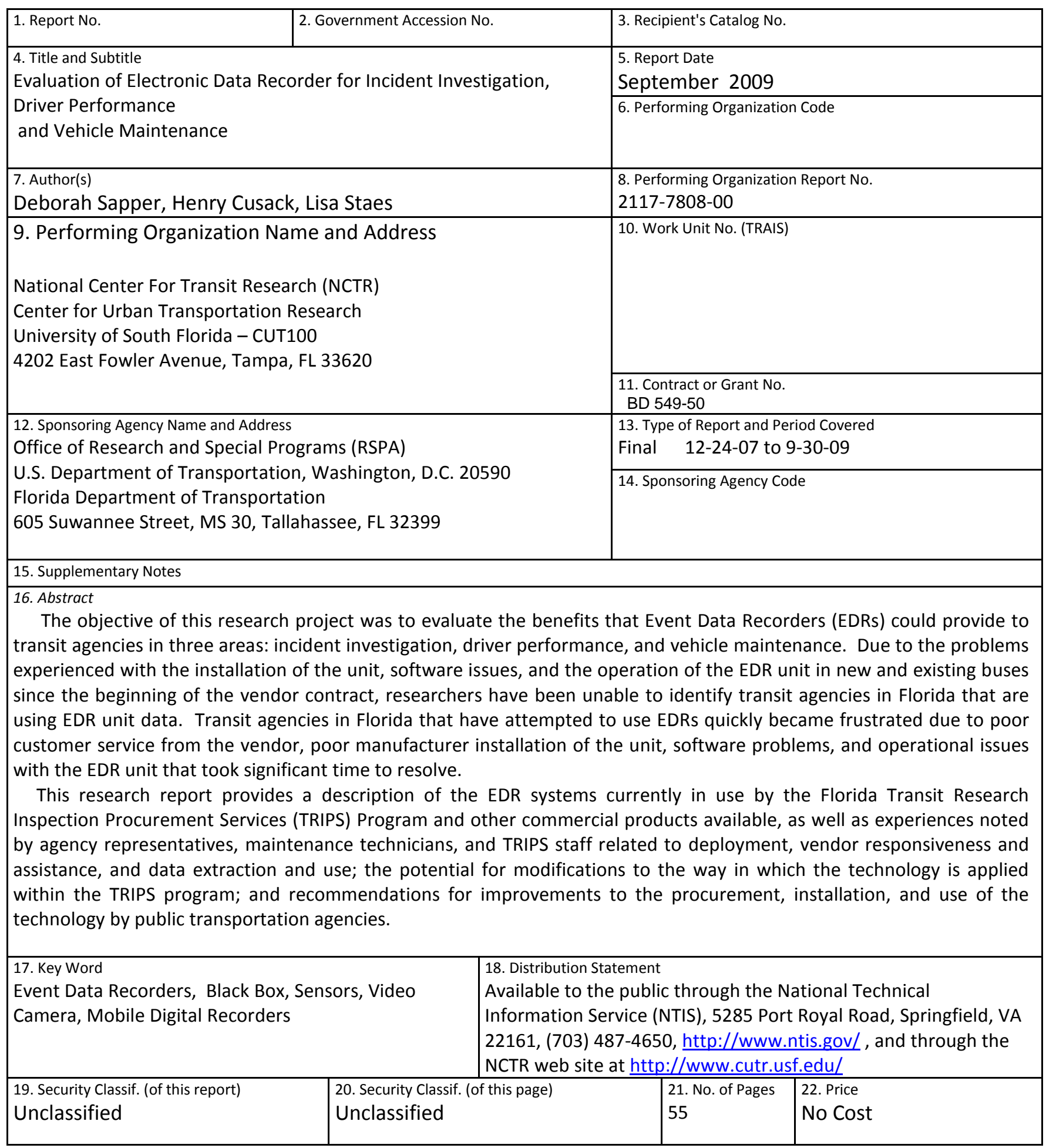




\section{EXECUTIVE SUMMARY}

Over the past few years, the number of commercial Event Data Recorders (EDRs) and Video Data Recorders (VDRs) available for use in public transportation has greatly increased. EDRs and VDRs are being used by transit agencies for accident investigation, driver performance, vehicle maintenance, and risk management. Systems such as AngelTrax, IDrive, Tacholink, DriveCam, Zepco, and 24/7 Security provide safety and security monitoring system by using a combination of video cameras, global positioning systems (GPS), and sensors.

The public transportation industry will benefit from the expanded use of EDRs in transit vehicles. EDRs/VDRs also will serve as valuable tools in minimizing frivolous lawsuits from passengers, pedestrians, and drivers of other vehicles. In addition, they may assist agencies in responding effectively to customer/rider complaints related to the safe operation of a vehicle or the behavior of a bus operator/driver.

Data from an EDR can assist agencies in monitoring bus operator performance and identifying training needs or remedial training. The data components most useful for these activities include g-force, fuel consumption, speed, hours driven, RPMs, heavy breaking, and fast acceleration. Tracking these items over time can provide clear indicators of poor or questionable performance and identify areas that may require additional or remedial training. Identifying performance issues and responding to them in a timely manner may prevent a collision, a passenger or operator injury, or damage to the vehicle.

This report looks at number of commercial EDRs and VDRs currently available for use by transit agencies. Detailed information is provided for Tacholink and 24/7 Security units, which currently are available for paratransit cutaway vehicles purchased through the Florida Transit Research Inspection Procurement Services (TRIPS) Program, with a brief overview of other EDRs and VDRs units currently available.

The objective of this research project was to evaluate the benefits that EDRs could provide to transit agencies in three areas: incident investigation, driver performance, and vehicle 
maintenance. Due to problems with the installation of the unit, software issues, and operation of the EDR unit in new and existing buses since the beginning of the vendor contract, researchers have been unable to identify transit agencies that are using EDR unit data. Transit agencies in Florida that have attempted to use EDRs quickly became frustrated due to poor customer service from the vendor, poor manufacturer installation of the unit, software problems, and operational issues with the EDR unit that have taken significant amount of time to resolve. Reliability, the data extraction process, and overall usefulness have been significantly less than expected. Unspecified installation procedures, lack of vendor oversight of manufacturers, failure to identify effects of changes to new chassis on EDR system operation, hardware design, and lack of end-user training are among the leading contributors to this lack of use and popularity among public transit systems.

This report considered installation problems, sensor errors, and other problems associated with EDRs installed on paratransit cutaway vehicles purchased through TRIPS, as reported by the component manufacturer field technician, and the corresponding "fix."

A major problem has been pre-wiring for sensors and cameras, which require pre-wiring during manufacturing of the passenger compartment upon the cab and chassis. It is imperative that the component manufacturer work closely with the original equipment manufacturer (OEM) and the second-stage manufacturer to ensure that an installation protocol is developed satisfactory to the vehicle manufacturers and component manufacturer. Vehicle manufacturers usually build the first bus as a prototype for testing, evaluation and inspection, and operation to conform to the technical specifications of the purchaser or customer contract. If the component manufacturer delivers the unit to the manufacturer without working with them on building the prototype, the component failure risk will be high and standardization of installation will not be identified and established for additional bus builds (which was the cause of some of the problems with the EDR units in Florida.)

Finally, the report looks at recommendations for improvements to the procurement, installation, and use of the technology by public transportation agencies. 


\section{TABLE OF CONTENTS}

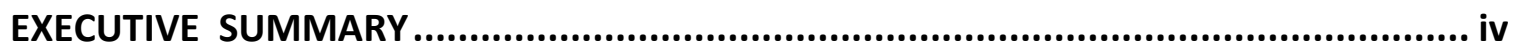

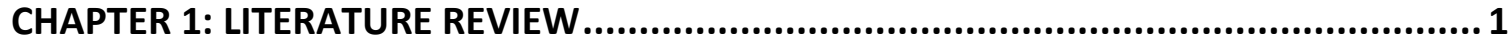

History of Commercial Vehicle Recorders ........................................... 5

EDR Research History .................................................................... 6

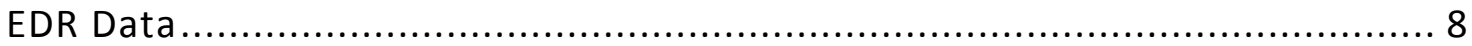

EDR Standards Groups................................................................ 13

Benefits of Collecting EDR Data ................................................... 17

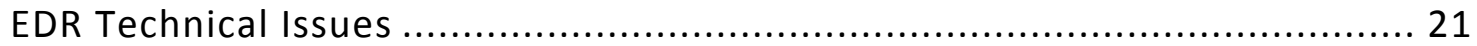

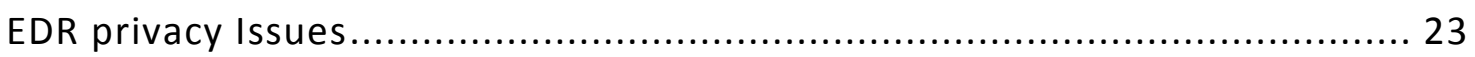

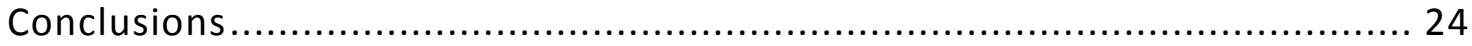

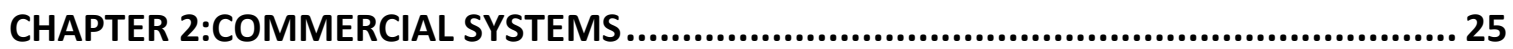

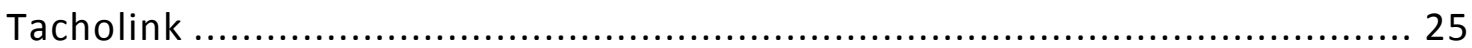

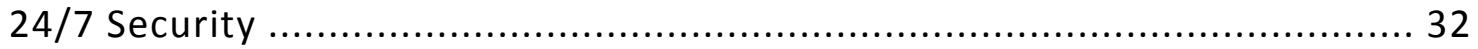

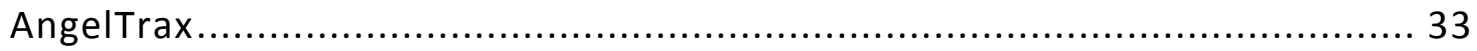

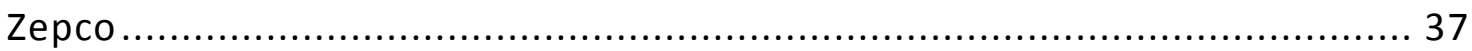

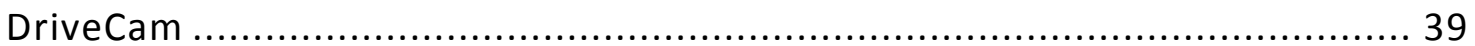

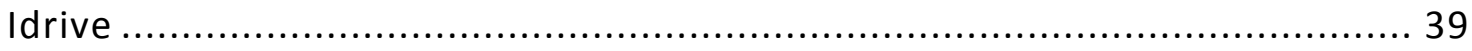

CHAPTER 3: PROBLEMS ASSOCIATED WITH MULTIPLE MANUFACTURER INSTALLATION OF EDR IN DIFFERENT OEM TRANSIT VEHICLES ........................................ 41

Component Installation Protocol ................................................... 41

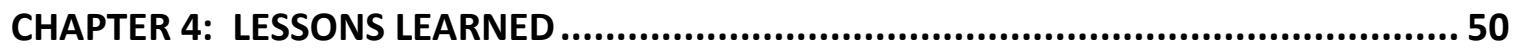

Recommendations.................................................................... 51

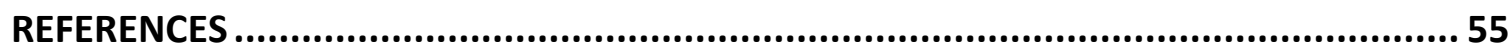




\section{LIST OF FIGURES}

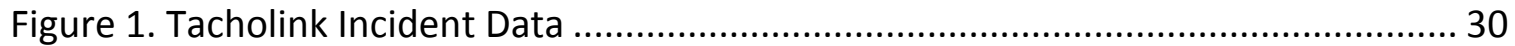

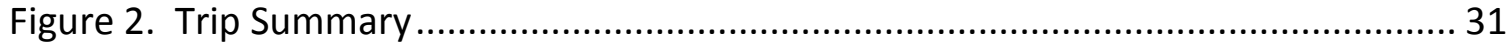

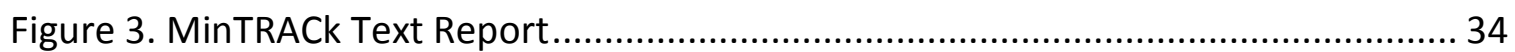

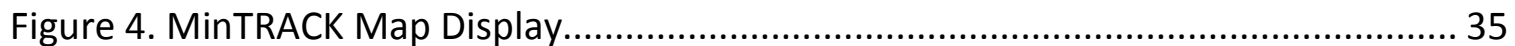

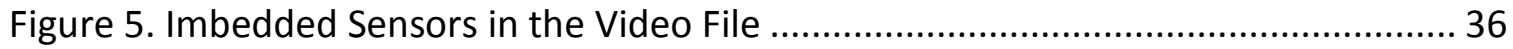

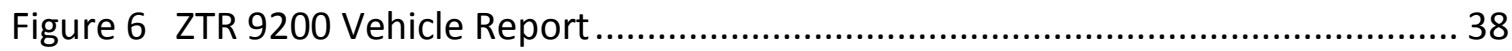

\section{LIST OF TABLES}

Table 1 Accident Reconstruction Issues. 


\section{CHAPTER ONE \\ LITERATURE REVIEW}

There have been several research efforts, studies, working group recommendations, and standards committee publications related to the installation, use, monitoring, data collection and extraction, and benefits of Event Data Recorders (EDRs) used in transportation including light rail, passenger vehicles, and transit buses. This literature review has captured publications released by the Society of Automotive Engineers, the Institute of Electrical and Electronics Engineers, the National Highway Traffic Safety Administration (NHTSA), and the National Cooperative Highway Research Program.

\section{Use of Event Data Recorder (EDR) Technology for Highway Crash Data Analysis NCHRP Project 17-24, December 2004}

The objectives of this research program included the development of recommendations for a minimum set of EDR data elements for roadside safety analysis and procedures for the retrieval, storage, and use of EDR data from vehicle crashes. This included a discussion of the legal and public acceptability of EDR use. NCHRP released the final report in December 2004 and as Web Document 75 in June 2005.

\section{NHTSA Light-Duty and Heavy-Duty Vehicle EDR Working Group Reports NHTSA, August 2001 and May 2002}

In 1998, NHTSA established the first EDR Working Group, which was tasked with the study of EDR technology, data elements, data retrieval, data collection and storage, permanent records, privacy and legal issues with the use of EDR data, the use of EDR data by consumers and other users, and the demonstrations of the technology. The first EDR Working Group published its findings in August 2001.

In 2000, NHTSA established a second working group to review those EDR-related issues associated with trucks, school buses, and motor coaches. They focused on data elements, accident survivability, and event definitions. The working group's findings were published in May 2002. 
Vehicle Event Data Interface - Vehicular Output Data Definition Society of Automotive Engineers (SAE), J1698 Standards Committee - J1698-1 Standard, SAE, December 2003

The SAE J1698 Standards Committee was established to develop common data output formats and definitions for those data elements that could be used for analyzing vehicle events, including accidents. The standard also specifies common connectors and network protocols to improve data extraction activities. In December 2003, the Vehicle Event Data Interface - Vehicular Output Data Definition (SAE J-1698-1 standard) was published by SAE.

\section{Motor Vehicle Event Data Recorders \\ Institute of Electronic and Electrical Engineers (IEEE), IEEE P1616 Standards Committee, February 2005}

The IEEE P1616 Standards Committee was established to define a protocol for motor vehicle event data recorder output data capability and export protocols for data elements. The committee established these protocols for both light- and heavy-duty vehicles. In February 2005, Motor Vehicle Event Data Recorders (IEEE P1616 standard) was published by IEEE.

\section{Development of Requirements and Functional Specifications for Event Data Recorders, FHWA IVI Program 134 Final Report} Federal Highway Administration (FHWA), December 2004

This report summarizes the work conducted by the NHTSA EDR Working Groups and associated EDR reference materials to define specific EDR requirements and specifications for the reconstruction of crashes involving large trucks (defined as those over 10,000 gross vehicle weight [GVW]). Included in the report are functional specifications for both complete crash reconstruction and less-detailed analyses of crashes. Requirements are defined for the various EDR components, hardware, software, sensors, and databases. This report also includes a cost-effectiveness analysis. In December 2004, the final report was issued by FHWA. 
Recommended Practice 1214 - Guidelines for Event Data Collection, Storage, and Retrieval

American Trucking Association (ATA) Technology and Maintenance Council

This recommended practice was issued by the ATA's Technology and Maintenance Council

to define the collection of event-related data available onboard commercial vehicles. It outlines data elements, storage methodology, and a retrieval approach; lists data parameters based upon message ID and parameter ID specifications of SAE J1587; and defines the recording interval as a period of 30 seconds before and 15 seconds after an event (an unspecified deceleration rate between 0 and $10 \mathrm{mph} / \mathrm{sec}$ ).

\section{A Review of Jurisprudence Regarding Event Data Recorders: Implications for the Access and Use of Data for Transport Canada Collision Investigation, Reconstruction, Road Safety Research and Regulation \\ Prepared for the Road Safety and Motor Vehicle Regulation Transport Canada, March 2005}

This report provides a brief introduction to the evolution of data recorders in passenger vehicles and other light-duty roadway vehicles, notes the increased availability and use of these systems, provides present and future benefits, and discusses the limitations of these systems in an effort to frame a discussion of jurisprudence with EDRs.

The primary focus of the report is to address the tension between balancing the many public benefits that are attributed to EDR technology and the appropriate protection of personal privacy. The report addresses the question of ownership of EDR units and the data that are generated from these units.

\section{Preliminary Evaluation of Advanced Air Bag Field Performance Using}

Event Data Recorders

H. Clay Gabler and Craig P. Thor (Virginia Tech) and John Hinch (NHTSA), August 2008, DOT HS 81101

This report describes a preliminary evaluation of the field performance of occupant restraint systems designed with advanced air bag features, including those specified in the Federal Motor Vehicle Safety Standard No. 208 for advanced air bags, through the use of event data recorders. Because EDRs record many of the inputs to the advanced air bag 
control module, these devices can provide unique insights into the characteristics of the field performance of air bags. This research program investigated the feasibility of using EDR data to evaluate advanced air bag systems. Specifically, this report discusses (1) the development of an expanded EDR dataset based on data retrieved from NASS/CDS 2005, $\mathrm{SCl}$, and $\mathrm{CIREN}$ in-depth crash investigations, (2) the validation of the accuracy of EDRs in full-scale crash tests, and (3) the feasibility of using EDRs to monitor the performance of advanced air bag restraints in real-world crashes.

\section{Analysis of Event Data Recorder Data for Vehicle Safety Improvement Marco P. daSilva, Volpe National Transportation Systems Center (VRTC), Cambridge, MA, April 2008, DOT HS 810935}

The Volpe Center performed a comprehensive engineering analysis of EDR data supplied by NHTSA to assess accuracy and usefulness in crash reconstruction and improvement of vehicle safety systems. The Volpe Center gathered and analyzed 2,541 EDR files downloaded from the National Automotive Sampling System (NASS), Special Crash Investigations $(\mathrm{SCI})$, and Crash Injury Research \& Engineering Network (CIREN) databases supplied by NHTSA. The analysis focused on EDR file format and potential improvements; assessment of crash types where EDR data exists; review of EDR data for accuracy and completeness; EDR data comparisons with existing crash data, review of pre-crash, crash, and post-crash data for usefulness in better understanding the crash reconstruction; identification of error sources; and determination of methods by which researchers could use the EDR data to improve their crash case information. The results of the engineering analysis showed that EDR data can objectively report real-world crash data and therefore be a powerful investigative and research tool by providing very useful information to crash "reconstructionists" and vehicle safety researchers. It was noted that, due to significant limitations, EDR data should always be used in conjunction with other data sources.

In addition to the research reports, committee standards, and associated publications, numerous technical papers and presentations have been developed around the issues central to the use of EDRs, the effectiveness of the data that are collected, the retrieval and use of the data, and other aspects of the technology and applications. 


\section{HISTORY OF COMMERCIAL VEHICLE RECORDERS}

The first EDRs or black boxes were used in the aviation industry in the late 1950s. In 1958, the Federal Aviation Act and corresponding regulations issued by the Civil Aeronautics Administration (the predecessor of the Federal Aviation Administration) made mandatory the use of black boxes or flight data recorders for commercial aircraft. In 1976, the National Transportation Safety Board (NTSB) issued regulations requiring the use of EDRs in commercial marine vehicles. In May 1995, the Federal Railroad Administration issued regulations requiring EDRs on heavy rail transportation. While the use of EDRs in automobiles and light trucks is currently voluntary, vehicle manufacturers such as General Motors and Ford have installed EDRs on many of their newer models.

The use of EDR technology in automobiles, buses, and small trucks began in the early 1980 s with the integration of computerized powertrain control systems and electronic transmissions. Engine and vehicle manufacturers recognized the opportunities to extract information from the onboard vehicle electronic systems and allow those systems to interact with one another. This developed into informational features extracted from powertrain control and electronic transmission systems that were displayed on the dashboard of the vehicle. In time, these data displays and associated computer systems evolved into recording devices.

At the same time, the evolution of computer-based vehicle systems was gaining momentum, and mobile radio, satellite, and cellular telephone based equipment was being introduced into commercial vehicles. This equipment was originally intended solely to assist with the routing and tracking of the vehicle to help with logistics management. Later, these communications systems became fully integrated trip recorders with many monitoring capabilities.

More recently, antilock braking systems, vehicle traction and stability controls, and airbag and restraint systems became standard on commercial vehicles of all sizes. Recording features have become part of these computer systems as well. In response, vehicle 
original equipment manufacturers (OEMs) recognized the importance of providing standardized data links on commercial vehicles. These links would enable data monitoring from these elements, as well as those generated by the computerized powertrain systems. Most vehicle OEMs now offer dashboard equipment and instrument clusters that read information off these data bus terminals. In some cases, this is recorded; in others, it is displayed only to the driver.

EDRs have made a major impact on highway safety and are being deployed as standard features on passenger vehicles, transit coaches/buses, light-duty trucks, and commercial vehicles. They are recognized as a valuable source of temporal data from immediately prior to immediately following a collision and can assist in accident reconstruction and litigation activities, determine causal factors, and even assist manufacturers in determining design factors that may have contributed to the severity of the impact and the injuries sustained by occupants (and respond to those factors by modifying design characteristics).

Recognizing the safety-related importance of EDRs, in 2002 the National Transportation Safety Board made a recommendation to the Federal Transit Administration (FTA) that new or rehabilitated buses funded through FTA grant program funds be equipped with EDRs. Since 2002, FTA has actively worked with heavy and light rail transit agencies to deploy EDRs. It is projected that by the year 2012, 50 percent of the rail systems within the United States will be equipped with EDRs.

The effort to deploy EDR technology on public transit buses and coaches is not as well organized. Few transit agencies within the United States have actively undertaken the deployment on transit buses. In contrast, in Florida, all public transit vehicles purchased with FTA Section 5310 funds, as well as those procured through the Florida Transit Research Inspection Procurement Services Program (TRIPS), are equipped with EDRs.

\section{EDR RESEARCH HISTORY}

Vehicle OEMs and component suppliers have studied EDR systems for many years. In 
addition, both publicly and privately funded research has been conducted. This research has focused on the various capabilities of the systems that were available at the time, the limitations of the systems and the retrieval of the data, and the benefits of using the systems for accident review and evaluation, vehicle system monitoring, and litigation activity, for example. Some of the most significant research activity has been conducted by NTSB and NHTSA. Many of these research documents and reports were highlighted in the literature review section of this document.

In 1997, NTSB issued recommendations to pursue the gathering of crash information using data extracted from EDRs. In April 1997, the National Aeronautics and Space Administration's (NASA) Jet Propulsion Laboratory (JPL) recommended that NHTSA "study the feasibility of installing and obtaining crash data for safety analyses from crash recorders on vehicles.

In early 1998, the NHTSA's Office of Research and Development launched a new effort to form a working group comprising industry, academia, and other government organizations. The EDR Working Group's primary objective was to facilitate the collection and use of collision avoidance and crashworthiness data from on-board EDRs. In August 2001, the Working Group published the results and findings of its efforts. This report included 29 findings that presented an overview from users to manufacturers.

In 2000, NHTSA sponsored a second working group that examined the use of EDRs based on the recommendations made by NTSB in 1999, specifically associated with trucks, school buses, and over-the-road coaches. Their objectives included the review of the specific data elements, occupant survivability, and event definitions. The findings were published in May 2002.

The research conducted by the working groups recognized the variability of output definitions and formats, communication protocols, and system connections that exists within the industry. In response, SAE established committees for the purpose of developing standards for these systems. A committee was also established by IEEE to 
research and define standards for output data compatibility and export protocols. ATA published a recommended practice to define the collection of event-related data onboard commercial vehicles. This includes the data elements collected, storage methodology, and the recommended retrieval approach.

NCHRP produced a report entitled Use of Event Data Recorder (EDR) Technology for Highway Crash and Data Analysis (NCHRP Project 17-24). The objectives of the research program included the development of recommendations for a minimum set of EDR data elements for roadside safety analysis and procedures for the retrieval, storage, and use of EDR data from vehicle crashes. This includes a discussion of the legal and public acceptability of EDR use.

While there are myriad examples of research and various studies related to the use of EDR data to assist in accident re-creation, the identification of causal factors, and the survivability of occupants, there is limited research on other aspects of EDR data use. Some of the important aspects or benefits to note are those related to increasing public transportation risk management and liability control; monitoring bus operator performance; identifying training needs or remedial training; investigating customer (rider) complaints; investigating allegations of misuse of equipment; reducing vehicle maintenance costs; diagnosing fleet defects; and other operational considerations.

\section{EDR DATA}

EDR devices record technical vehicle and occupant-based information for a brief period of time (seconds, not minutes) before, during, and after a crash. For instance, EDRs may record pre-crash vehicle dynamics and system status, driver inputs, vehicle crash signature, restraint usage/deployment status, and certain post-crash data such as the activation of an automatic collision notification (ACN) system.

The way this is accomplished can be described in the following somewhat simplified manner. The EDR monitors several of the vehicle's systems, such as speed, brakes, and 
several safety systems. It continuously records and erases information on these systems so that a record of the most recent, such as an eight-second period is always available. If an event occurs (i.e., a crash meeting a predetermined threshold of severity), then the EDR moves the last eight seconds of pre-crash information into its long-term memory. In addition, it records and puts into its long-term memory up to six seconds of data relating to what happened after the start of the crash, such as the timing and manner of deployment of the air bags. The degree of benefit from EDRs is directly related to the number of vehicles operating with an EDR and the current infrastructure's ability to use and assimilate these data.

The data collected by the EDR can be maintained within the device for approximately 60 days for non-deployment services, but can be stored permanently for deployment events (accidents). In existing EDR systems, a subsequent serious accident or other event can erase the data. A decoder can be used to make it easier to download data from the scene of an accident.

NHTSA has recognized the benefit of using EDR data in activities related to accident reconstruction and the determination of causal factors. As such, the agency has been using EDR data to support its crash investigation program for several years. The various data parameters logged by the EDR as described by NHTSA are as follows:

- Ignition cycle crash - the number (count) of power cycles applied to the recording device at the time the crash event occurred since the first use of the EDR.

- Ignition cycle download -the number (count) of power cycles applied to the recording device at the time the data were downloaded since the first use of the EDR.

- Lateral acceleration - the component of the vector acceleration of a point in the vehicle in the $y$-direction. The lateral acceleration is positive from left to right from the perspective of the driver when seated in the vehicle facing the direction of forward vehicle travel. 
- Longitudinal acceleration -the component of the vector acceleration of a point in the vehicle in the $x$-direction. The longitudinal acceleration is positive in the direction of forward vehicle travel.

- Maximum delta-V, lateral - the maximum value of the cumulative change in velocity, as recorded by the EDR, of the vehicle along the lateral axis, starting from crash time zero and ending at 0.3 seconds.

- Maximum delta-V, longitudinal - the maximum value of the cumulative change in velocity, as recorded by the EDR, of the vehicle along the longitudinal axis, starting from crash time zero and ending at 0.3 seconds.

- Multi-event crash - the occurrence of two events, which begin no more than 5 seconds apart.

- Non-volatile memory - the memory reserved for maintaining recorded EDR data in a semi-permanent fashion. Data recorded in non-volatile memory is retained after a loss of power and can be retrieved with EDR data extraction tools and methods.

- Normal acceleration- the component of the vector acceleration of a point in the vehicle in the z-direction. The normal acceleration is positive in a downward direction and is zero when the accelerometer is at rest.

- Occupant position classification - the classification indicating that the seating posture of a front outboard occupant (both driver and right-front passenger) is determined as being out-of-position.

- Occupant size classification - for the right-front passenger, the classification of an occupant as an adult and not a child, and for the driver, the classification of the driver as not being of small stature.

- Pretensioner - a device activated by a vehicle's crash sensing system that removes slack from a vehicle safety belt system. 
- Safety belt status - the feedback from the safety system that is used to determine than an occupant's safety belt (for both driver and right-front passenger) is fastened or not fastened.

- Seat track position switch, foremost, status - the status of the switch installed to detect whether the seat is moved to a forward position.

- Service brake, on and off - the status of the device installed in or connected to the brake pedal system to detect whether the pedal was pressed. The device can include the brake pedal switch or other driver-operated service brake control.

- Side air bag - any inflatable occupant restraint device mounted to the seat or side structure of the vehicle interior and designed to deploy in a side-impact crash to help mitigate occupant injury and/or ejection.

- Side curtain/tube air bag -any inflatable occupant restraint device is mounted to the side structure of the vehicle interior and designed to deploy in a side-impact crash or rollover and to help mitigate occupant injury and/or ejection.

- Speed, vehicle indicated - the vehicle speed indicated by a manufacturerdesignated subsystem designed to indicate the vehicle's ground travel speed during vehicle operation.

- Stability control -any device not directly controlled by the operator (e.g., steering or brakes); intended to prevent loss of vehicle control by sensing, interpreting, and adjusting a vehicle's driving and handling characteristics and controlling or assisting the driver in controlling the vehicle.

- Steering wheel angle - the angular displacement of the steering wheel measured from the straight-ahead position (position corresponding to zero average steer angle of a pair of steered wheels). 
- Suppression switch status - the status of the switch indicating whether an air bag suppression system is on or off.

- Time from event $\mathbf{1}$ to $\mathbf{2}$ - the elapsed time from time zero of the first event to time zero of the second event.

- Time, maximum delta-V, longitudinal - the time from crash time zero to the point where the maximum value of the cumulative change in velocity is found, as recorded by the EDR, along the longitudinal axis.

- Time to pretensioner - the elapsed time from crash time zero to the deployment command for the safety belt pretensioner (for both driver and right front passenger).

- Time to deploy, side air bag/curtain - the elapsed time from crash time zero to the deployment command for a side air bag or a side curtain/tube air bag (for both driver and right front passenger).

- Time to first stage - the elapsed time between time zero and the time when the first stage of a frontal air bag is commanded to fire.

- Time to maximum delta-V, lateral -time from crash time zero to the point where the maximum value of the cumulative change in velocity is found, as recorded by the EDR, along the lateral axis.

- Time to nth stage - the elapsed time from the crash time zero to the deployment command for the nth stage of a frontal air bag (for both driver and right front passenger).

- Time zero - for systems with "wake-up" air bag control systems, the time occupant restraint control algorithm is activated; for continuously running algorithms, the first point in the interval where a longitudinal, cumulative delta- $V$ of over $0.8 \mathrm{~km} / \mathrm{h}$ (0.5 $\mathrm{mph}$ ) is reached within a $20 \mathrm{~ms}$ time period; or for vehicles that record "delta- 
$\mathrm{V}$, lateral," the first point in the interval where a lateral, cumulative delta- $\mathrm{V}$ of over $0.8 \mathrm{~km} / \mathrm{h}(0.5 \mathrm{mph})$ is reached within a $5 \mathrm{~ms}$ time period.

- Trigger threshold - a change in vehicle velocity, in the longitudinal direction, that equals or exceeds $8 \mathrm{~km} / \mathrm{h}$ within a $150 \mathrm{~ms}$ interval. For vehicles that record "delta$\mathrm{V}$, lateral," trigger threshold means a change in vehicle velocity, in either the longitudinal or lateral direction that equals or exceeds $8 \mathrm{~km} / \mathrm{h}$ within a $150 \mathrm{~ms}$ interval.

- Vehicle roll angle - the angle between the vehicle $y$-axis and the ground plane.

- Volatile memory - the memory reserved for buffering of captured EDR data. The memory is not capable of retaining data in a semi-permanent fashion. Data captured in a volatile memory are continuously overwritten and not retained in the event of a power loss or retrievable with EDR data extraction tools.

- X-direction - the direction of the vehicle X-axis, which is parallel to the vehicle's longitudinal center line. The $\mathrm{X}$-direction is positive in the direction of forward vehicle travel.

- Y-direction - the direction of the vehicle $\mathrm{Y}$-axis, which is perpendicular to its $\mathrm{X}$-axis and in the same horizontal plane as that axis. The Y-direction is positive from left to right from the perspective of the driver when seated in the vehicle facing the direction of forward vehicle travel.

- Z-direction - the direction of the vehicle Z-axis, which is perpendicular to the X-and $\mathrm{Y}$-axes. The Z-direction is positive in a downward direction.

\section{EDR STANDARDS GROUPS}

Historically, EDR designs have been developed independently by each automaker to meet their own vehicle-specific needs. There has not been a common format or protocol for data collection, retrieval, or maintenance. There is tremendous variation in the data elements 
and the definition of these data element. For example, both General Motors and Ford Motor Company record vehicle impact response versus time or crash pulse. General Motors stores the crash response as a velocity-time history recorded every 10 milliseconds; Ford stores the crash response as an acceleration-time history recorded every 0.8 millisecond. This lack of standardization led to national-level studies of vehicle and roadside crash safety and the establishment of EDR standards working groups. There are working groups established within several organizations that represent the interests of commercial and passenger vehicles, as well as a working group established by the American Public Transportation Association (APTA) that is specifically developing recommendations and standards for transit buses.

Currently, three professional organizations are actively developing standards for vehicle EDRs used in commercial and passenger vehicles. These groups are defining industry standards or recommended practices for EDR formatting, methods of retrieval, and procedures for record archiving. The three active working groups include:

(1) IEEE P1616 Standards Working Group on Motor Vehicle Event Data Recorders

(2) Society of Automotive Engineers (SAE) J1698 Standards Working Group on Vehicle Event Data Interfaces; J1939 Working Group on serial data bus communication standards; SAE J2728 Commercial Vehicle Event Data Recorder Standards Committee

(3) ISO/TC22/SC12/WG7 group on Traffic Accident Analysis Methodology

The accomplishments of each group and the status of ongoing efforts are summarized below:

- IEEE 1616 - IEEE Standards Association (IEEE-SA)

The IEEE 1616 Standards Association approved the IEEE 1616 standard for Motor Vehicle Event Data Recorders (MVEDRs) in September 2004. The IEEE 1616 standard defines a minimum standard for onboard crash recorders for all types of 
highway vehicles including passenger cars, light trucks, heavy trucks, and buses. In January 2002, the IEEE P1616 Working Group began to concentrate on the standardization of both candidate EDR data elements and the EDR output connector. The standard now includes a data dictionary of 86 data elements. It does not specify a minimum set of data elements but provides a standardized definition for individual data elements. The IEEE 1616 group is following up this effort with IEEE P1616a, "Standard for Motor Vehicle Event Data Recorders (MVEDRs) - Amendment 1: Brake and Electronic Control Unit (ECU) Electronic Fault Code Data Elements."

- SAE J1698 - Society of Automotive Engineers (SAE)

The scope of the SAE J1698 standards development effort was to develop common data output formats and definitions for a variety of data elements that may be used for analyzing vehicle "events," most notably crashes. The standards are intended to govern data element definitions and data extraction methodology. Further, the standard will specify common connectors and network communications protocols to facilitate the extraction of such data. In December 2003, SAE J1698-1 was issued, providing a recommended practice for a Vehicle Event Data Interface (VEDI). The J1698-recommended practice applies only to passenger cars and light trucks. The VEDI committee has strong industry support for this standard, as indicated by very active participation from the automakers.

- SAE J1939 - Society of Automotive Engineers (SAE)

The scope of the SAE J1939 standards development effort was to develop a serial data bus communication standard for truck, bus, off-road, construction and marine vehicle applications. The $\mathrm{J} 1939$ communication standard is a control and information data bus that supports critical safety-related systems and subsystems. Safety critical systems that are currently in production that use this standard 
include engines, transmissions, drive slip control (part of the anti-lock braking system), collision avoidance, and land guidance systems.

- SAE J2728 - Society of Automotive Engineers (SAE)

The scope of the SAE J2728 standards development effort is to "establish common data elements and data element definitions for heavy commercial vehicle event data recording." The committee is dealing specifically with crash event data recording rather than vehicle data logging and recording. The goal of the committee is to develop a standard that specifies event triggers, threshold levels, and survivability. The committee will also recommend procedures for data extraction.

- ISO/TC22/SC12/WG7 - International Organization for Standardization (ISO)

The scope of the ISO/TC22/SC12/WG is to establish standardized methodologies for traffic accident analysis. The use of EDR technologies and the various data that can be extracted from EDRs is under review by this working group.

In the area of public transportation, APTA has recognized the value of establishing standards for EDRs and has established a Vehicle Data Recorder (VDR) Working Group. The current focus of the group is the development of a recommended practice for vehicle data recorders on transit buses. The overall purpose of the group is to provide guidance to both suppliers and transit agencies in the performance requirements of electrical components or system of components that will meet the needs of the nation's transit systems. The VDR Working Group is attempting to balance the regulations and requirements of NHSTA for event data recorders with the actual data elements that will assist transit agencies improve daily operations. 


\section{BENEFITS OF COLLECTING EDR DATA}

The collection and use of EDR data has many benefits. Most research conducted to date tends to focus on the benefits related to accident reconstruction, causation, and litigation support. However, there are ongoing operational and maintenance-related benefits that can be obtained with the use of EDR data. For public transportation agencies, the use of EDR data can increase public transportation risk management and liability control, assist agencies monitor bus operator performance, identify training needs or remedial training, investigate customer (rider) complaints, investigate allegations of misuse of equipment, reduce vehicle maintenance costs, and diagnose fleet defects, as well as other operational considerations.

\section{ACCIDENT RECONSTRUCTION AND INVESTIGATION}

The benefit of EDR data for state transportation agencies is improved investigation of individual crashes, including providing compelling evidence toward the identification of causal factors and identifying the role of human error. EDR data are increasingly being used to reconstruct aspects of a crash, such as vehicle speed immediately before a crash and at impact. EDRs can provide more complete and accurate information than thorough crash reconstruction aided by current simulation software and vehicle dynamics theory. Many state and local law enforcement organizations already collect EDR data on a regular basis for fatal crash investigations. EDR data can be a powerful form of evidentiary support in legal proceedings.

EDR data can make the difference in resolving key issues of causation and fault. For example, recent EDR data retrieved after an intersection collision established that one of the vehicles involved in the collision had been traveling nearly twice the speed limit approximately five seconds before the crash. Further calculations demonstrated that the collision could have been avoided if the vehicle had been traveling at a more reasonable speed. As a result, the driver who was speeding was found primarily liable for the crash. The EDR information was crucial to this investigation because, although the driver had 
braked heavily, the antilock brake system in his car prevented skid marks (a traditional source of evidence of speed) from appearing on the road. The EDR provided the only evidence of excessive speed.

In another case, event data downloaded from a vehicle that had collided with a telephone pole indicated that the driver of the vehicle had pushed the gas pedal to the floor for about three seconds and accelerated to more than $60 \mathrm{mph}$ just before impact. The data supported witness reports that the vehicle was racing another vehicle before the crash. In addition, the event data indicated that the driver was not wearing his seatbelt at the time of the crash, contributing to the severity of his injuries.

The relevance and usefulness of EDR data is not limited to high-speed collisions. EDR data can be used to identify causes and conditions and indicate operator errors in less severe, non-injury accidents and accidents involving pedestrians, those that occur in parking areas, and those that occur within congested corridors. Table 1 summarizes typical accident reconstruction issues related to EDR data.

\section{Table 1 Accident Reconstruction Issues}

\begin{tabular}{|l|ll|}
\hline \multicolumn{1}{|c|}{ Issues } & \multicolumn{1}{c|}{ Crash Data } \\
\hline Liability and Fraud & - $\begin{array}{l}\text { Pre-crash vehicle speed brake status percent of throttle } \\
\text { and steering wheel angle }\end{array}$ \\
\hline Airbag Performance & - & Airbag status at impact and deployment timing details \\
\hline Injury & - Impact severity \\
\hline Seat Belt & - Impact severity \\
\hline
\end{tabular}

\section{STATISTICAL ANALYSIS AND RESEARCH}

EDR data can significantly improve the efficiency of data collection for crash statistic databases. The use of EDR data can improve the accuracy of these databases and may reduce the long-term costs of more labor-intensive data collection and retrieval.

One of the crucial long-term benefits of EDRs will be their influence on highway crash safety research. The ready availability of EDR data in a crash statistics database will enable 
vehicle and roadside safety researchers to address several elusive and often technically controversial research questions, including the relevance of impact conditions for roadside crashes, if there is a link between vehicle acceleration and occupant injury, if current vehicle designs are compatible with existing roadway safety hardware designs, and if advanced occupant restraint systems performing as designed.

\section{BENEFITS FOR THE PUBLIC TRANSPORTATION INDUSTRY}

The public transportation industry will benefit from the expanded use of EDRs in transit vehicles. The use of EDR technology, including video data recording, and the data that can be extracted from these units may lead to increased public transportation risk management and liability control for the agency. As mentioned previously, EDR data are being used in the courtroom to provide detailed information of the conditions that existed immediately prior to, during, and immediately following a collision. They may also serve as a valuable tool in minimizing frivolous lawsuits from passengers, pedestrians, and drivers of other vehicles. They may lead to reduced Workman's Compensation claims. In addition, they may assist agencies effectively respond to customer/rider complaints related to the safe operation of a vehicle or the behavior of a bus operator/driver. They may lead to reduced customer complaints if unsafe drivers are identified during routine driver performance reviews.

The data may assist agencies in monitoring bus operator performance and identifying training needs or remedial training. The data components most useful for these activities include fuel consumption, speed, hours driven, RPMs, heavy breaking, use of turn signals and four-way hazard flashers, and fast acceleration. Tracking these items over time can provide clear indicators of poor or questionable performance and identify areas that may require additional or remedial training. Identifying these performance issues and responding to them in a timely manner may prevent a collision, a passenger or operator injury, or damage to the vehicle. 
There are also benefits related to the maintenance of the vehicles, satisfactory continuing control, and the identification of fleet defects. Vehicle maintenance-related data indicators include fuel sensors, lights, handbrake engagement, air conditioning systems, water temperature, oil pressure, fuel consumption, speed, and RPMs. Data elements that can be tracked that may provide valuable data to maintenance technicians include heavy braking and fast acceleration events and idle time. Data may provide trend information for preventative maintenance activities. Information may also indicate automatic vehicle location system/GPS download issues or systematic issues with onboard computers. A recent example of the benefit of an EDR to fleet maintenance is when a new bus was delivered to Florida with the right front wheel significantly out of alignment. The driver claimed it was fine when dropped off. The EDR was checked and it was determined the right front had hit something hard. The data provided the date and time of the incident, which enabled a check of that road and a deep pothole at that location was found

\section{LIMITATIONS OF DATA RECORDED BY EDRS}

Event data recorders do have limitations, including the following:

- Most new vehicles do not have advanced EDRs, but rather recorders that capture only data from airbag crash sensors and may not gather other information such as pre-crash speed and safety belt usage.

- Some EDRs restrict data retrieval to the maker of the vehicle.

- There are circumstances where an air bag deployment command would be issued but the algorithm used to order the deployment determines a deployment is not warranted, such as a driver out of position before deployment is ordered.

- Data may be recorded for non-deployment events such as rollover, sideswipe, and side impact accidents.

- It is also possible that no data can be recovered from a data recorder. One situation where this might occur would be a catastrophic loss of electrical power during the 
collision. In this situation, the entire power reserve in the air bag control module capacitor is used to deploy the air bags, leaving none for the recorder. Examination of data save to that point may provide a hint at what caused the electrical failure.

\section{EDR TECHNICAL ISSUES}

One technical issue of concern regarding the use of EDR is the data elements that should be collected. Some of the more common data elements include crash pulse information, safety belt use, air bag deployment status, pre-crash data, and vehicle identification number. It is also important that these data elements are technically and economically feasible. Also, some organizations believe that there should be different set of data for light duty vehicles and heavy trucks. Another technical issue that needs to be addressed is the amount of data to be logged or recorded. Generally, EDRs collect data for a very brief period of time (up to 10 seconds for pre-crash and post-crash data and several 10ths of a second for crash data).

Another technical issue is related to the packaging, storage, and various uses of EDR data. The protocol for these issues must be defined and fully communicated to the end-user. If this does not occur, the user to have little confidence in the data regardless of the product vendor, the sensors that have been activated, the use of cameras, or other configurations, even if the unit is functioning effectively. Vendor training must be provided for the enduser to effectively manage the maintenance of the system, access and use the data, download software, and troubleshoot when necessary.

There are also technical issues related to the installation of the EDR units, the various methods employed by OEMs to allow connection of the EDR units to the onboard electrical systems, the pre-wiring during the manufacture of the passenger compartment in transit cutaway (body on chassis) vehicles, and the procedures/protocols used to extract data.

There are issues related to the communication between component manufacturers, OEMs, and second stage manufacturers related to installation protocol. These entities must work 
together to ensure a consistent installation protocol is developed. The TRIPS program recorded a number of technical issues that are listed below and discussed more thoroughly in the following chapters of this report.

Major errors due to a lack of installation protocol include the following:

1. EDRs were operating 24 hours per day, 7 days per week. This resulted in some OEM ECM systems never shutting down and draining the batteries. Agencies that experienced this found that over extended periods of time out of service (over a weekend), the vehicle batteries were dead.

2. A standard configuration and thresholds for the EDR based on customer parameters were not initially established, resulting in excessive, inconsistent data warnings between individual buses and fleets.

3. Monitoring speed and RPM were difficult without OEM authorization to connect to the OEM ECM system computer. In this example, the use of GPS provided the best resolution..

4. The wiring harnesses provided by component manufacturers were not uniform, creating problems with the EDR sensor's positive connection.

5. The type of sensor wires used and the manner in which the wiring was placed within the vehicle walls and along the chassis created poor connections. For many TRIPS vehicles, this resulted in the wheel chair lift sensor requiring frequent adjustment to properly monitor the lift operation.

The TRIPS program continues to monitor and respond to technical issues with the EDR units installed on paratransit cutaway vehicles procured through the program. Additional discussion of these issues and the solutions that have been implemented to alleviate them will be discussed in the sections that follow. 


\section{EDR PRIVACY ISSUES}

There are obvious safety benefits with EDRs. However, the use of EDR data, "ownership" of the data, and other associated privacy issues have been topics of controversy. Privacy concerns seem to be particularly problematic for those advocating the general use of EDRs. However, more support is being garnered in the interest of public safety versus the underriding case for individual personal property rights and what is viewed as an unwarranted intrusion. Many consumers may not even be aware that EDRs are installed in their vehicles. They also may not realize that the data collected by these devices may be used not only to aid in traffic safety analysis, but potentially used against them in civil or criminal proceedings, or by their insurer. While these are valid concerns, there appear to be overriding benefits to public safety that prevents significant scrutiny. In A Review of Jurisprudence Regarding Event Data Recorders: Implications for the Access and Use of Data for Transport Canada Collision Investigation, Reconstruction, Road Safety Research, and Regulation, prepared for the Road Safety and Motor Vehicle Regulation, Transport Canada, it was noted that "evolving jurisprudence in both criminal and civil jurisdictions appears to significantly limit the owner to a reasonable expectation of privacy in allowing access to this data by many interested parties."

Efforts are under way to ensure that EDR crash-related data used in research activities does not contain personal information that would reveal the identity of individuals involved in the collisions. Recognizing that the encryption of data and the use of associated security codes may be an alternative, it is noted that standards concerning this encryption and access must be established.

Beyond the controversy surrounding the use and admissibility of EDR data from personal vehicles, there could also be privacy concerns from drivers of commercial vehicles and those driving public transportation vehicles. If EDR data are used to monitor behavior and performance indicators, particularly when this information leads to remedial training, penalty, or dismissal of a driver, maintenance technician, or other employee, it could 
become problematic. Collective bargaining units may determine that this monitoring activity violates current bargaining unit and employer agreements.

As the integration of these systems become more prevalent in the commercial vehicle and public transportation industries, there is the potential for additional concerns and protests to be identified.

\section{CONCLUSIONS}

While the widespread deployment of EDRs offers a new and unique glimpse of the events that occur during a highway traffic collision, that are equally beneficial elements in the public transportation industry that are not related to collisions. As more public transportation agencies make the decision to implement EDR technologies on transit buses and other vehicles, it will be important to have the tools available to determine the technologies available. Learning from the experiences of those that have deployed this technology will be critical.

The balance of this research report provides a description of the EDR systems currently in use by the Florida TRIPS Program; the experiences noted by agency representatives, maintenance technicians, and TRIPS staff related to deployment, vendor responsiveness and assistance, and data extraction and usage; the potential for modifications to the way in which the technology is applied within the TRIPS program; and recommendations for improvements to the procurement, installation, and use of the technology by public transportation agencies. 
CHAPTER 2

COMMERCIAL SYSTEMS

Over the past few years, the number of commercial EDRs and VDRs available for use in public transportation has greatly increased. EDRs and VDRs are increasingly being used by transit agencies for accident investigation, driver performance, vehicle maintenance and risk management. System such as AngelTrax, Idrive, Tacholink, DrivCam, Zepco, and 24/7 Security provide safety and security systems monitoring by using a combination of video cameras, global positioning systems (GPS), and sensors.

The J1939 CAN bus interface allows a simple "plug \& play" installation on any compliant vehicle and five external connections for other devices or system monitoring. This unit can be used for monitoring speed and engine, seat belt use, directional signals, brake operation, light activation, emergency lights, horn operation, door operation, or any system that is electrically operated or can be fitted with a micro switch.

This section of the report looks at number of commercial EDRs and VDRs currently available for use by transit agencies. Detailed information is provided for the Tacholink and 24/7 Security units, which are currently available for vehicles purchased through the Florida TRIPS program, with a brief overview of other EDRs and VDRs units that are currently available.

\section{TACHOLINK}

Listed below are the requirements for the Tacholink EDR units from vehicle contract FVPP05-CA-1 for vehicles purchase under the Florida TRIPS Program, along with additional information on the Tacholink unit.

2.45.2.1 An event data recorder (EDR), Circuitlink International "Tacholink", (or approved equal) will be mounted on the vehicle. 
2.45.2.2 The EDR will provide a continuous log of vehicle activity. Speed history, odometer, excessive RPM, heavy braking, fast acceleration, idling exceptions will automatically recorded by exception.

2.45.2.3 A three axis accelerometer shall be provided to provide a log of excessive movement in any direction. In the event of an accident, all data shall be permanently captured in "tamperproof" flash memory (4MB minimum) for a minimum of (30) seconds prior to the incident and (15) seconds after the incident at a minimum interval of 25 milliseconds.

2.45.2.4 The EDR will provide an automatic trigger function with different user definable thresholds for moving and stationary conditions.

2.45.2.5 A manual driver alert button will be provided to allow driver "tagging" of either an accident or vehicle fault condition shall be located behind upper bulkhead and must be accessible through a latching door.

2.45.2.6 Additional digital input channels will be provided to allow for status monitoring of a maximum of eight (8) vehicle sub-systems. Standard systems that will be monitored shall be: brakes, lights, signals, flashers, driver's seat belt, wheelchair lift, engine temperature and front door. Each agency may substitute any of the standard systems detailed above with any of the following optional systems: oil pressure, low air pressure, low voltage, or emergency exit door. The limit of the total systems to be monitored is eight (8).

2.45.2.7 Any data provided by the EDR must be admissible in court.

2.45.2.8 All software for user configuration, data logging, and downloading and report generation will be included. All software will be Windows-based. Trip data will be stored in Microsoft Access database. All data will be the property of Florida DOT and will be immediately available to Florida DOT. 


\section{ADDITIONAL INFORMATION ON THE TACHOLINK EDR}

An EDR has been factory-installed on a bus as a tool to assist the Florida Department of Transportation in reconstructing and analyzing unusual "events" while the bus is in operation. These events might include traffic accidents, vehicle dynamic conditions, subsystem activity, bus performance, etc. The EDR may be used as a passive device where all data are accumulated for long periods of time and accessed only in the event of an unusual occurrence.

The EDR unit also may be used as an active fleet management tool should a transit authority choose to do so. The EDR unit is continuously recording a large amount of data about how the bus is being operated, driver performance, and driving habits, and all actions and reactions are stored in permanent memory for recall at any time.

There are a number of fleet management tools available for use with the EDR.

\section{DRIVER INTERFACE}

The Driver Interface is a "Smart Tag" button, worn on a keyring, and allows an operator to "log in" to the bus. The onboard EDR unit monitors and records all bus and operator activity for each individual driver and trip. When completing a work shift, the driver "logs out." At that time, all information, including hours of service, is downloaded to the operator's individual "Smart Tag." A total of 250 sessions may be stored on the tag. This option requires one Smart Tag button for each driver. At any desired frequency, transit management may chose to "download" the accumulated data from each tag for analysis and/or review with the operator.

\section{HISTORIC BUS LOCATION \& MAPPING (GPS RECEIVER)}

The Historic GPS Receiver allows the Tacholink to continuously record vehicle time and location (latitude and longitude) at whatever frequency the transit operator may choose. This data may be downloaded at any time for review and analysis. Detailed map overlays for both Florida and appropriate regional areas are provided. Typical items analyzed may 
be route activity, schedule compliance, call response times, vehicle location, idling and/or inactivity time and location, etc.

\section{DATA DOWNLOAD INTERFACES}

If the unit is not being used as a fleet management tool, all data can be conveniently downloaded to a standard laptop computer. The Digital Smart Module (DSM) is a permanent memory device that allows for easy download of data from multiple vehicles for transfer to any standard Windows computer for review and analysis

If transit management chooses to fully automate the data downloading each time a bus returns to the depot, a Radio Frequency (RF) modem system can be installed. The RF system automatically polls each bus equipped with an RF sending unit and transfers the data to a standard Windows computer. Specific data elements to be downloaded are userselectable.

In normal recording mode, the EDR stores acquired data on a minute-by-minute basis, thus providing a readily accessible data string for average speed - distance - time, which can be readily used by fleet managers to compare vehicle/driver performance.

\section{FLEET MANAGEMENT}

The more fuel used, the more the vehicle costs to run. Information about fuel consumption can be especially useful when comparing different drivers and vehicles. By looking at the fleet management data, a driver's profile becomes clear. By allowing the fleet manager the freedom to set event profiles to automatically record what are considered exceptions to normal driving, a daily log is produced. The exceptions can be for exceeding predetermined static or idle times with the engine running and speed or acceleration/deceleration values. 


\section{ACCIDENT INVESTIGATION / RECONSTRUCTION}

The EDR unit can be programmed to automatically store a higher level of data required for accident investigation. The trigger threshold is set to a deceleration value, which indicates an impact or other incident. Once the unit recognizes that an incident has occurred, it stores all data from 45 seconds prior to the incident until the vehicle comes to rest, or for 20 seconds after the incident, whichever is longer.

By reviewing data held in the instrument, it is possible to determine in great deal exactly what a driver or vehicle was doing for up to 45 seconds prior to the incident. This information comprises vehicle speed, engine speed, lateral and longitudinal accelerations, (braking/accelerating/swerving), operation of vehicle equipment and signals. etc. All information is recorded in $25 \mathrm{~m} / \mathrm{sec}$ increments and distance.

\section{GRAPH OF INCIDENT DATA}

Figure 1 is an example of a basic Tacholink graph of incident in which a bus rear-ended another vehicle during rush hour traffic. The operator claimed the brakes did not work properly. As can be seen from the incident data in Figure 1, the vehicle was traveling at 50 mph. The operator hit the brakes 1.6 seconds before impact. In that 1.6 seconds, the vehicle speed decreased to $36 \mathrm{mph}$ at impact, indicating that the brakes were working properly.

Figure 2 indicates that the operator often was speeding ( 7 instances of $75+\mathrm{mph}$ ).

Using this data, the transit agency was able to clearly demonstrate that the vehicle was in good working order and the operator was clearly at fault. 

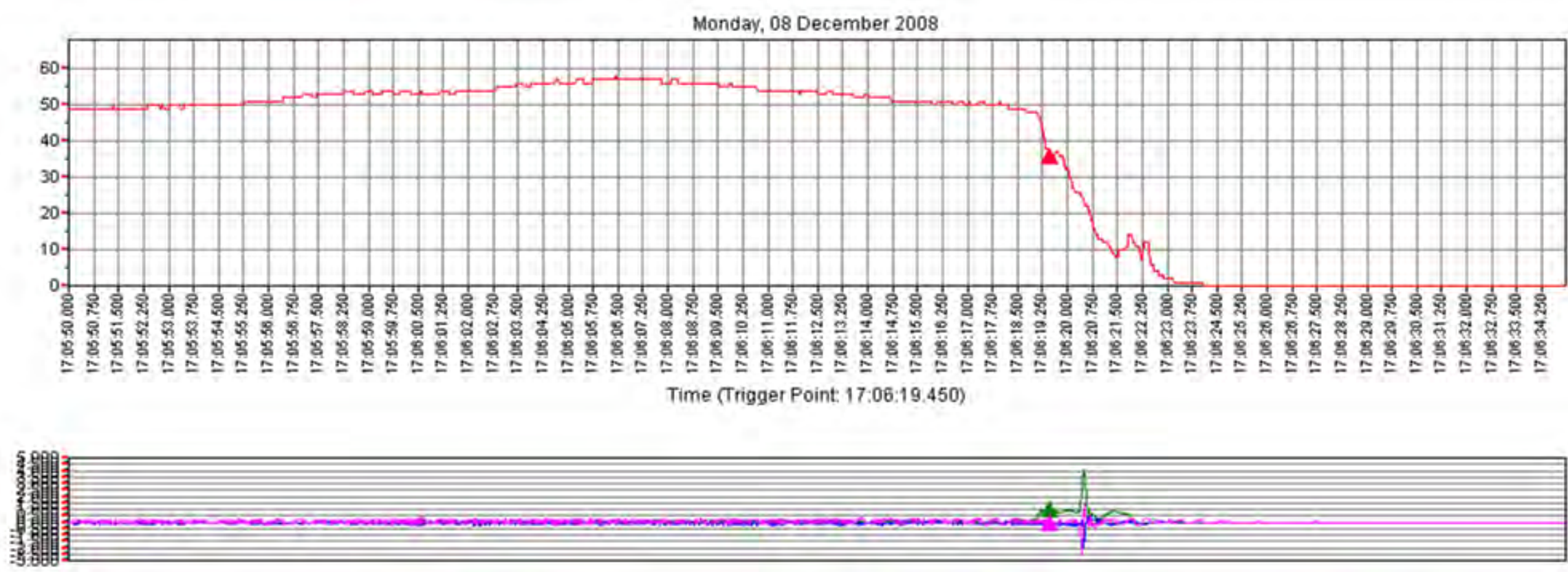

Figure 1. Tacholink Incident Data 


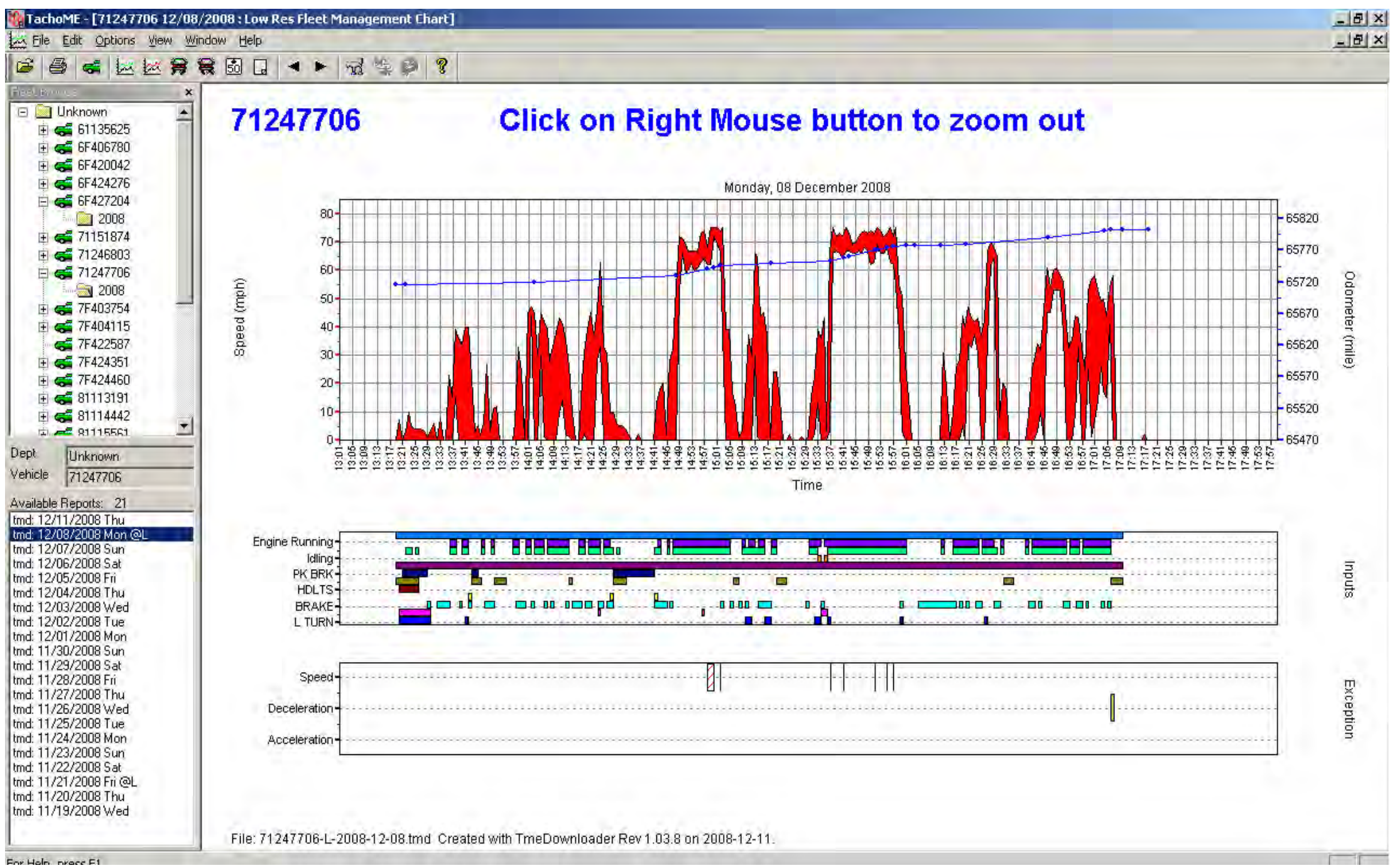

Figure 2. Trip Summary

Additional information about Tacholink can be found at http://www.tttonline.com/product/edr/edr.htm 


\section{4/7 SECURITY}

The 24/7 Security unit combines camera surveillance and sensors into the base unit Mobile Digital Recorder (mDVR), with an accelerometer module added to collect g-forces and provide input for eights sensors. The base unit is a digital video recorder with an "all-in-one" system to serve data collection requirements and provide ultra high quality video, audio, and vehicle tracking features. Some of the features and capabilities of the mDVR include:

- Standard mapping and routing features, which are valuable management tools to improve operations, lower cost, and provide assistance for driver training.

- Speed detection is not connected to the vehicle computer and uses an independent input source, which is less intrusive and more reliable.

- Data retrieval is done through the download to a flash drive in a few seconds.

- Data can be collected from dozens of vehicles on a single flash drive and reviewed when convenient.

- As agencies recognize the necessity of video recording for liability, driver training, and vehicle maintenance purposes, they will not need to buy two different systems for each vehicle.

- Better product, better customer service and training, more useful features, simpler operation, and more reliable data.

- High resolution video $(720 \times 480)$ for sharp images and dedicated audio recording channel for each video channel.

The MiniTRACK GPS Management System continuously tracks and monitors vital vehicle information. The MiniTRACK GPS unit has advanced data compression that allows storage of up to 1 gigabyte of trip and sensor data. Vehicle reports are available through the Add Record button, which allows users to upload GPS data collected from the vehicle to the MiniTRACK 
data base. The MiniTRACK GPS tracks idling, stops, speed, impact, event markers, and g-force by occurrence by user-defined parameters. Once the data have been uploaded, reports are available for any period of time, anywhere from one day to one year, and data can easily be exported to other software applications for review and analysis. Figure 3 is an example of a MiniTRACK text report. The MiniTRACK GIS map displays each occurrence of user-defined routes, stops, parked vehicles, idling, speeding, impacts and event marker. Figure 4 is example of the MiniTRACK GIS map.

The mDVR On-Screen-Display (OSD) embeds the sensor name into video files. When playing back the video, the sensor(s) can be identified by the embedded text on the video. PreRecording (in seconds) and Post-Recording (in seconds) also can be predefined in the sensor trigger recording process. Figure 5 is an example of the embedded sensors in the video file. The video file displays date and time, the speed of the vehicle, if the headlights are on, and if the brake has been applied.

The 24/7 Security mDVR unit is available with the video cameras and MiniTRACK GPS Management Systems or the MiniTRACK System without the cameras. Additional information on the $24 / 7$ Security unit can be found at http://www.247securityinc.com/index2.html

\section{ANGELTRAX}

The AngelTrax Hybrid component is a mobile DVR that is field serviceable. The system comes with either a 4-channel or 8-channel camera and audio input. Once installed, any repair, upgrade or hard drive retrieval can be accomplished without removing the DVR unit. The unit has eight sensor inputs for marking events such as left turn, right turn, brake, lights, doors, gforce sensors, and speed. The unit comes with a passive GPS or Virtual Synchronized Mapping (VSM) that provides on-screen route tracking to identify movements of the vehicle on a digital street map, synchronized with the cameras views and recorded to the DVR. Data can be searched by time, date, or event. Data can be transferred to a USB 2.0 Memory Key for marked-event viewing or through a wireless connection. Additional information on AngelTrax can be found at http://www.angeltrax.com/ 


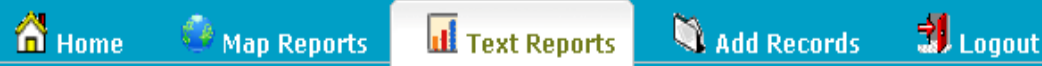

utr-dsappe

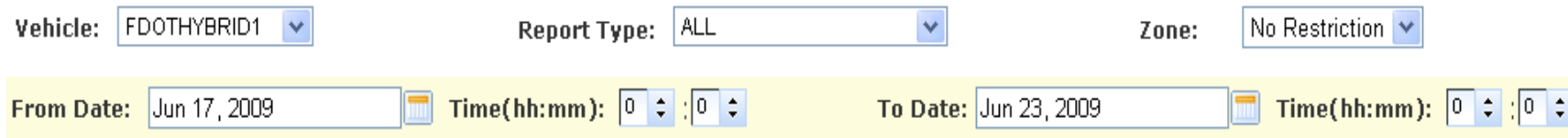

GENERATE

ALL Report

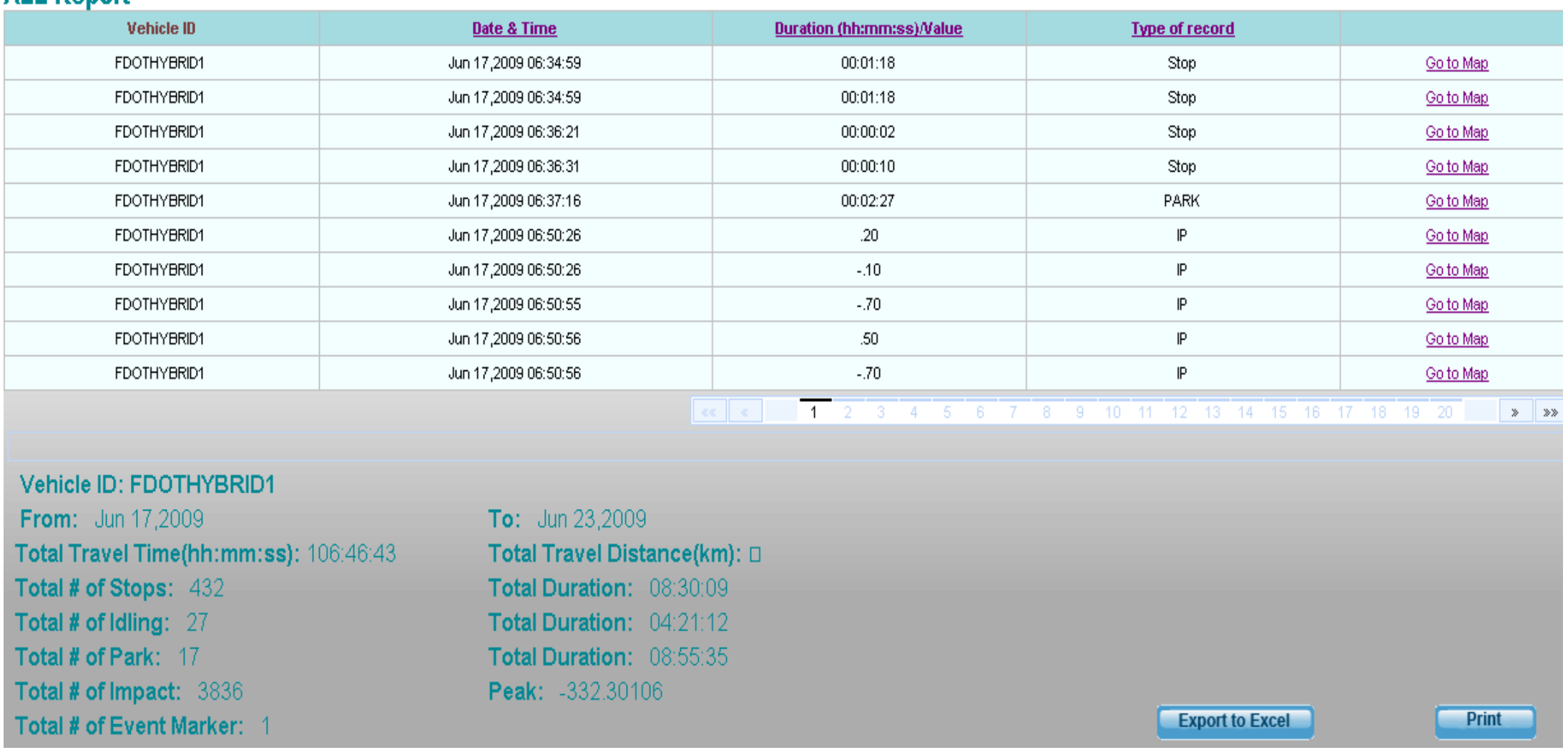

Figure 3. MinTRACK Text Report 


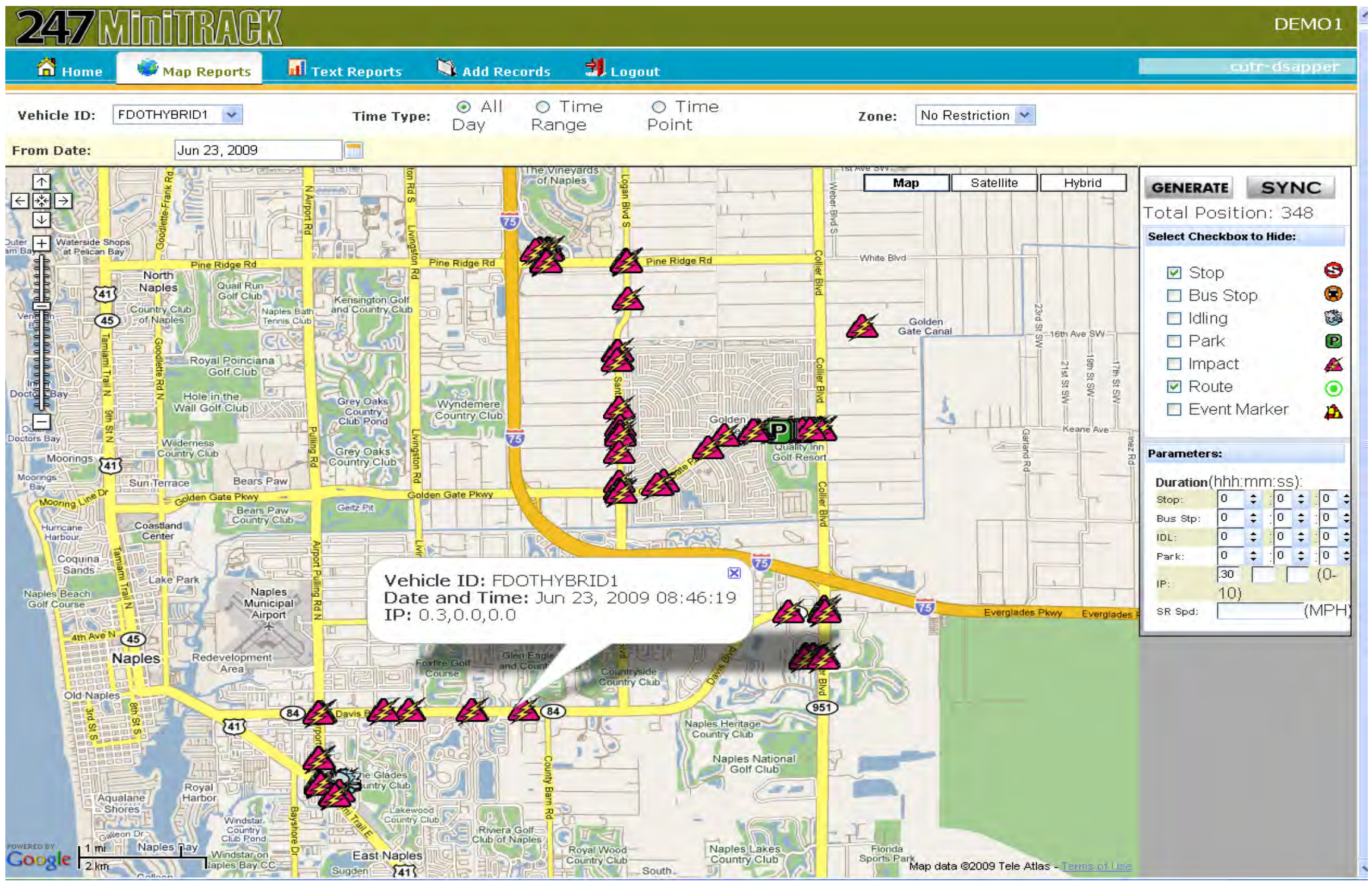

Figure 4. MiniTRACK Map Display 


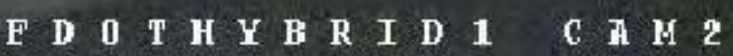

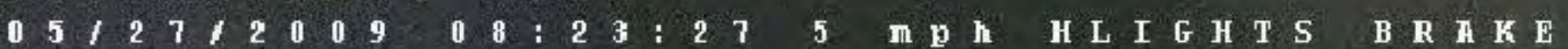

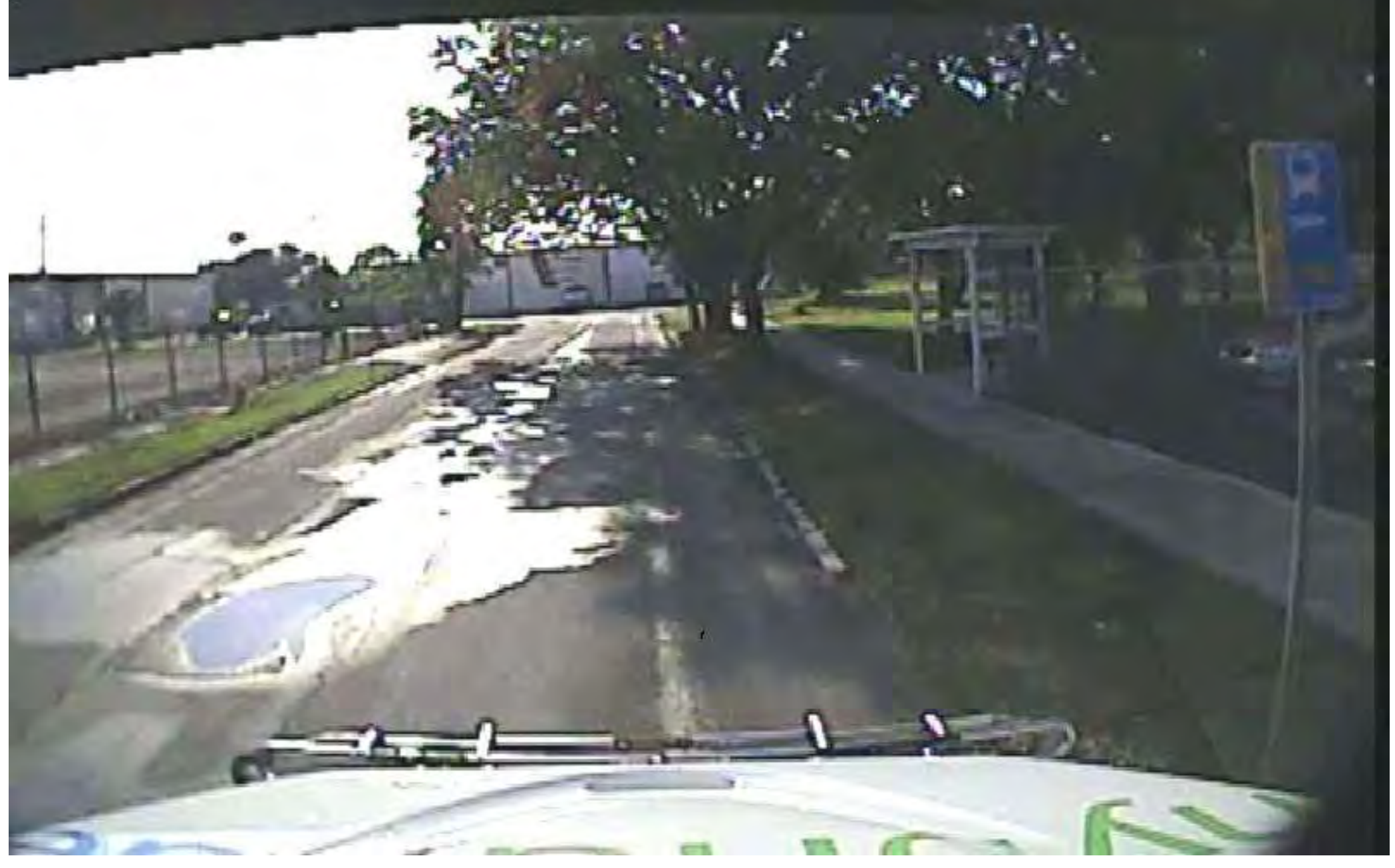

Figure 5. Imbedded Sensors in the Video File 
The 24/7 Security mDVR unit is available with the video cameras, sensors and MiniTRACK GPS Management Systems or the MiniTRACK System, sensors displayed on the mDVR unit but without the cameras. Additional information on $24 / 7$ Security can be found at http://www.247securityinc.com/index2.html

\section{ZEPCO}

The ZR4 is a full digital recorder with simultaneous video and audio channels and a storage capacity 80 to $160 \mathrm{~GB}$. The unit can record to an SD flash memory or to the computer hard drive. The ZR4 also records GPS and offers up eight input sensors customized to the user needs, such as speed, impact, and location etc. Data can be downloaded through a video connection using the module's video-out connectors and on-board software by removing the module's removable hard drive and connecting it through the USB port to a desktop or laptop computer or via wireless download.

The Zepco ZTR9200 vehicle data logger tracks idle time, hard braking, and speeding with the option to monitor up 11 other system inputs that affect fleet operating cost. The unit runs on an Intel microprocessor, and data are accessed through Windows. The ZTR9200 provides summary easy-to-read reports for all inputs that are being tracked. Figure 6 is example of a summary report from the ZTR 9200 data recorder. Additional information on ZEPCO can be found at http://www.zepco.com/. 


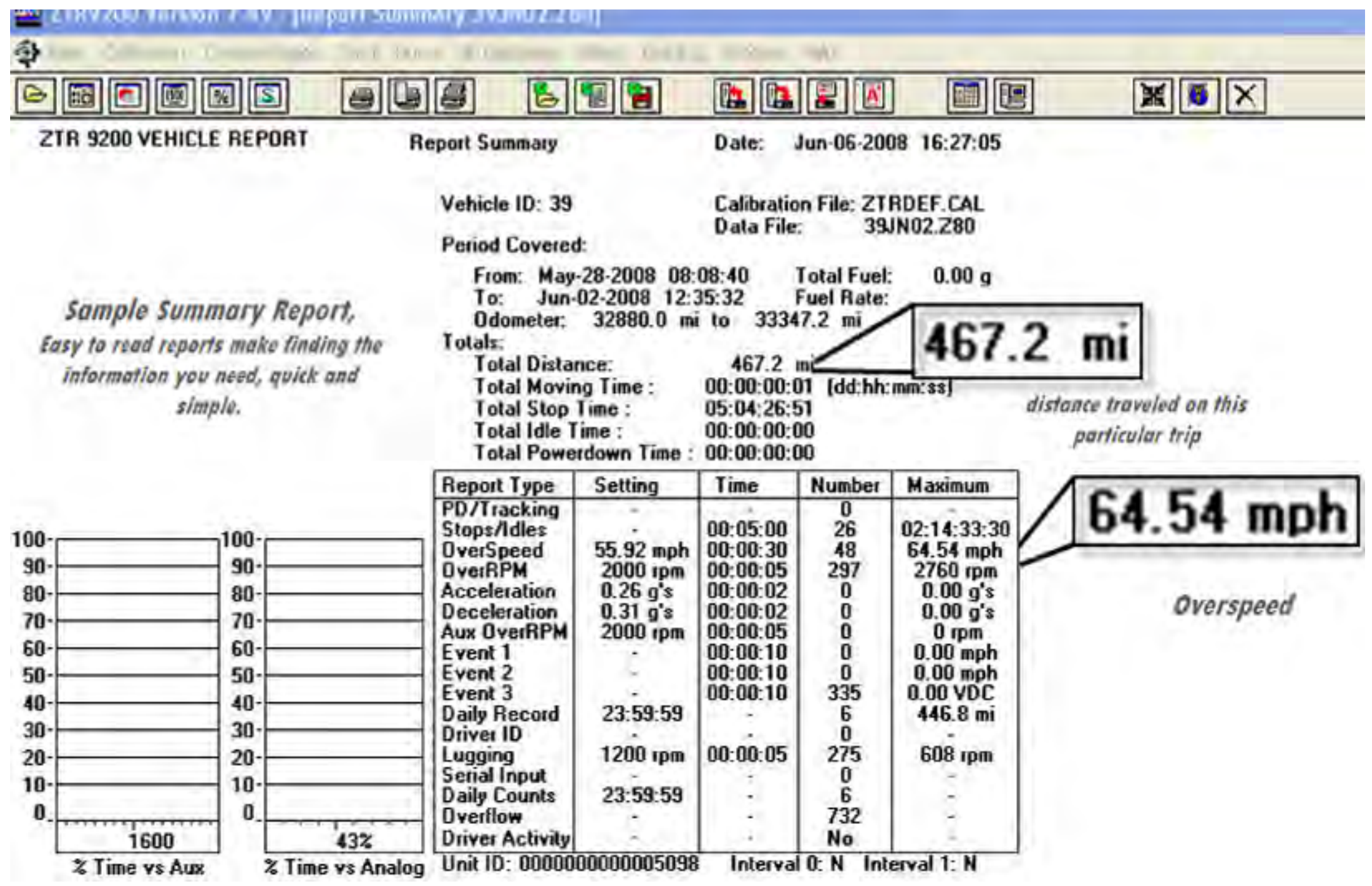

Figure 6 ZTR 9200 Vehicle Report 


\section{DRIVECAM}

DriveCam's Driver Risk Management identifies risky driving behaviors that pose a threat to the safety of drivers, passengers, and other motorists and provides immediate feedback on driver performance. The behavior-based service incorporates audio and video event capture, expert analysis, and driver coaching. Additional information on DriveCam can be found at http://www.drivecam.com. Listed below are features of the DriveCam system:

- The DriveCam is placed on the windshield with two cameras, one near the driver's face and the other with a forward view. It records accelerometers on the $\mathrm{y}_{-}, \mathrm{x}-$-, and $\mathrm{z}$-axis in constant loop g-forces $\geq 0.5$. DriveCam captures events 8 seconds before and 4 seconds after event. Event-based data includes speed, location, and heading via GPS.

- The software service platform consist of online interface, compact interactive reporting, user dashboards, and advanced event viewing and coaching information screens based on Microsoft's advanced Silverlight ${ }^{\mathrm{TM}}$ player.

- Reports detail fleet driving improvement and areas of focus; results are benchmarked and provide insight into program effectiveness.

- Certified Driving Risk Analysts can review events on a daily basis and deliver timely feedback about drivers to fleet managers, ensuring a consistent coaching methodology across the organization and maximizing cost savings.

- Wi-Fi or cellular download options are available.

\section{IDRIVE}

Idrive is a professional EDR that can capture high definition video from the front of the vehicle and back with dual wide-screen angle lenses. The video is stored in internal memory along with data from the accelerometer, sensors, and the GPS. The GPS receiver records location coordinates and speed information when the tracking system service is active. 
The idrive unit can help detect aggressive driving behavior, accidents, speed, and exceptional forces such as hard braking, swerving, and collision. The recorder saves audio and video of the event immediately before and after the event was triggered. Data can automatic be downloaded to a computer or server through a wireless network or through a USB adaptor when there is no wireless network available.

The idrive software is managed by web-based software that synchronizes the video with other data. The software provides detailed vehicle safety and vehicle diagnostics data to fleet managers to identify bad driving behavior. Additional information on idrive can be found at http://idrive.pro/ 


\section{CHAPTER 3 \\ PROBLEMS ASSOCIATED WITH MULTIPLE MANUFACTURER \\ INSTALLATION OF EDR IN DIFFERENT OEM TRANSIT VEHICLES}

This chapter reviews installation and operational problems associated with EDRs on vehicles purchased through the new transit vehicle contract of FDOT's TRIPS Program. EDR units have been a standard component on paratransit cutaway transit vehicles procured under the program for the past seven years. The data from the EDR units is primarily intended to be used to support FDOT's bus rollover and side impact research of cutaway buses. The TRIPS Program has multiple new public transit vehicle contracts available from which Florida transit agencies can choose, with an EDR system standard in most contracts.

The original scope of this project was to look at how EDRs could be used in transit accident investigation analysis. Since 2006, TRIPS contracted with a different EDR vendor manufacturer for a newer-generation product that provides additional features that not only enhance accident investigation but also customer damage/accident claims investigation, as well as assisting in diagnosing maintenance issues and evaluating driver performance. Due to problems associated with the installation of the unit, software issues, and the operation of the EDR unit in new and existing buses since the beginning of this contract, researchers have been unable to indentify transit agencies in Florida that are using EDR unit data. Some transit agencies in Florida attempted to use the EDRs, but quickly became frustrated due to poor customer service from the vendor, poor manufacturer installation of the unit, software problems, and operational issues that took significant time to resolve.

\section{COMPONENT INSTALLATION PROTOCOL}

As with any new component, the specific bus criteria will determine the manner of installation and configuration of the component. Fixed-route "purpose built" bus requirements are similar to those for cutaway buses for the component manufacturer. A cutaway bus (a passenger compartment built by a second stage manufacturer on an OEM chassis and cab) has additional challenges for those components that need to be connected to the OEM ECM system. 
An EDR installed on public transit buses is available with three basic options: a lone g-force crash impact data unit, a recorder with up to 12 sensors monitoring the operation of key vehicle- and operator-actuated systems, and independent camera/audio systems. The EDR can be any combination of the three basic units. Use of camera systems can reduce the number of sensors required. Whether installing sensors, cameras, or a combination of the two, all require pre-wiring during manufacturing of the passenger compartment on the cab and chassis. It is imperative that the component manufacturer work closely with the OEM and the second stage manufacturer to ensure that an installation protocol is developed satisfactory to the vehicle manufacturers and component manufacturer. Vehicle manufacturers usually build the first bus as a prototype for testing, evaluation and inspection, and operation to conform to the technical specification of the purchaser or customer contract. If the component manufacturer delivers the unit to the manufacturer without working with them on building the prototype, the component failure risk will be high, and standardization of installation will not be identified and established for additional bus builds (which was the cause of some of the problems with the EDR units in Florida.) Each chassis manufacturer type (Ford, GMC, International, etc.) should have the same protocol no matter which second stage manufacturer is building the vehicle.

It is imperative that the purchaser or customer understand the operating features of a new component beyond the normal manufacturer "sales pitch." This can be time-consuming and requires an expert who may not be available in-house to represent the purchaser.

Listed below are errors indicated from the EDRs installed in vehicles purchased through TRIPS, as reported by the component manufacturer field technician, and the corresponding "fix":

\section{DEAD BATTERIES}

- Dead batteries at Sarasota County Area Transit and at Clay County Council of Aging were believed to be related to the EDR, but no specified defect was identified, but disconnecting the EDR unit power source resolved the dead battery issues. 
- On four buses, the backup brake pump motor is powered and runs with the EDR connected. When the ignition is off, with the EDR unit disconnected, this does not happen.

- Chevrolet Uplander Minivans had problems with dead batteries, particularly after two or more days of inactivity. After disconnecting the EDR unit, fuse batteries would hold a charge. The EDR AMP draw on the battery was identified as not strong enough to drain the charge from the battery. However, it was determined that with the EDR sensors connected to the headlights, turn signals, brake lights, etc., the OEM body engine computer modual (ECM) continued operating when the ignition was in the Off position. The battery power required to keep the EDR and the OEM control module active for more than 36 hours was calculated to exceed the battery capacity.

FIX: The power connection for the EDR was installed to be operating 24/7. This resulted in the OEM chassis computers never powering down. The standard power connection was changed to "ignition on" for activation of the EDR system, which resolved the dead battery issues.

\section{EDR PIN HARNESS, PLACEMENT, AND WIRING PROBLEMS}

- The following example was primarily a function of the EDR component vendor not working closely with vehicle manufacturers to standardize installation and certify that the system is working properly before each bus departs the factory for delivery to the purchaser: the vehicle manufacturer installed a 12-pin harness and 1-wire-in-2-pin harness instead of using a 15-pin harness with 2 unused pins. The 1-pin harness was not connected and several wires from the 12-pin were torn out, which resulted in a unit that could not communicate.

FIX: Standardized to a 15-pin harness, re-crimped new pins and put them in the new pin connector. 
- A 2009 bus had the wrong EDR-recorded miles and no RPMs. When checking the wiring of the harnesses and speed filters, it was found that the speed and RPM wires in the vehicle harness were reversed.

- The hotwire on the vehicle harness side was crimped to an unstripped wire. The EDR registered 0 miles while the bus had over 50,000 OEM miles.

- Factory placement of EDR units used the standard 20-pin harness supplied with the units instead of cutting it off and re-pinning it to a 15-pin harness.

- Factory installed the EDR unit as far back as possible in the cavity of the driver overhead compartment without providing an access opening. It was impossible to download data or repair or change the unit. To access the EDR unit, the manufacturer developed a jig to cut an access hole in the panel behind the driver and covered it with a standard panel cover for consistency on similar buses. A technician would simply need to remove the access panel cover to get to the EDR unit. This procedure was then applied on the assembly line to all new builds.

- No ground identified to the dead EDR unit. The EDR connection was located under the rubber floor mat directly behind the driver's seat. The technician could barely reach the connection without removing the seat. The technician was not equipped to remove the seat and could barely plug in the speed filter. Some of the insulation was missing from the harness side of the speed filter. The ground wire was severed and stripped back slightly. It was connected with a wire nut to a 14-gauge green wire, which was then run to the grounding strip in the compartment above the driver. The floor panel had a thick coating of paint, making a ground difficult to ensure. The connection was wrapped in rubber tape. The client was advised to have the seat removed and have a proper ground made before the EDR harness was connected. 
FIX: The EDR vendor assured that the system to be installed in the buses were "plug \& play," which was not the case. Installation required the splicing of wires and a installation protocol was developed for plant.

To fix the problem the manufacturers are now providing a plug to access the harness. Each manufacturer differs in the type of connection and type of wiring used, which results in different programming parameters. The EDR vendor needs to work more closely with each manufacturer to ensure each chassis type installation is identical, regardless of the second stage manufacturer. The EDR component vendor needs to work closely with manufacturers when chassis are revised or upgraded to determine affects on their system and necessary revisions to their "as-built" specification.

\section{CANNOT DOWNLOAD CONFIGURATION}

- The EDR unit cannot download the configuration with the idle exception buzzer on.

- EDR unit cannot download data.

FIX: The EDR technician identified a defective EDR box or outdated software version.

\section{EDR MILES ARE INCORRECT}

TRIPS staff observed during the vehicle inspection that EDR miles are incorrect; the miles on the EDR are 1,288, while the OEM miles are 1,136. Other examples include:

- EDR miles are incorrect: EDR miles 1,660, OEM miles 1,098,

- EDR miles are incorrect: EDR miles 1,501, OEM miles 1,061.

- EDR miles are incorrect: EDR miles 131, OEM miles 1,069.

- Recording speed at idle, EDR miles are incorrect.

FIX: The EDR incorrect speed problem was corrected by adding a speed pulse divider on the GMC 3500 chassis and a speed filter on GMC $4500 / 5500$ chassis. This was a very common 
problem found during post-delivery inspections at the FDOT testing facility in Tallahassee. The vendor should have investigated the possible impact on their EDR system due to changes in the introduction of the new OEM chassis instead of reacting to problems after installation. Use of GPS is another resolution to this problem, but adds to the EDR cost.

\section{OEM GAUGES "GOING CRAZY”}

- The OEM gauges were reported as "going crazy" when technicians attempted to connect diagnostic computers. The problem ceased when the EDR was disconnected. The technicians were not sure this issue was specific to certain manufactured vehicles, but when they disconnected the EDR unit, the issue was resolved.

FIX: This was corrected by adding a speed pulse divider on the Chevy 3500 chassis and a speed filter on the Chevy 4500 and 5500 chassis.

\section{NOT RECORDING RPM}

- The EDR units were not recording RPM.

FIX: The tracking of RPMs was discontinued as it never was accurate and no fix was identified by the EDR manufacturer. No known OEM computer access location was available to connect for data retrieval. Monitoring speed and RPM were difficult without OEM authorization to connect to the OEM computer. GPS seemed to be the best resolution, but is an additional cost from the vendor.

\section{SENSOR RECORDING ERRORS}

- EDR unit was not recording parking brake.

- EDR units were recording "brake applied" when the brake was not applied.

- EDR units were not recording entry door and wheel chair lift operation.

- EDR speed wire was not connected. 
FIX: This issue was directly related to poor installation due to lack of "as-built" specifications. The development and implementation of these standards resolved most sensor operation problems. PM follow-up repairs made by the transit agencies continue to be an issue with sensors going out of adjustment or alignment, or losing contact.

\section{INOPERATIVE SENSORS}

- $\quad$ EDR unit indicated left and right turn signal inputs were inoperative.

- $\quad$ Lift stow input inoperative.

- Headlights input inoperative.

- Door input inoperative

- $\quad$ Lift stow input inoperative

FIX: Inoperative input means that the input is not triggering the logged status and will not tag when activated. The agencies are directed to call their bus dealer to correct the problem. Normally, these issues are related to the manufacturing process. The sensors that trigger these inputs are manufacturer warranty issues. The EDR vendor provides the unit, harness, sensors and wiring, but all are installed by the second stage manufacturer. The EDR vendor does not run wiring throughout the bus for sensors. The wheel chair lift sensor continues to require frequent adjustment to properly monitor the lift operation. The vendor feels confident these issues are related primarily to out-of-adjustment lift/stow switches. The use of a camera with a date/time stamp would provide more information.

\section{ERROR MESSAGE WHILE TRYING TO UPDATE SOFTWARE}

- A bad chip was found in the EDR unit.

FIX: The EDR unit needed to be replaced. The unit should have been checked before leaving the factory, which would have identified this issue before delivery. 


\section{ATX CONNECTION}

- Factory configuration does not match FDOT configuration.

FIX: The EDR vendor specifies the installation and EDR unit configuration to meet with manufacturer and purchaser requirements. Configuration on the EDR unit should have been checked before leaving the factory.

\section{LACK OF A STANDARD CONFIGURATION AND THRESHOLDS FOR THE EDR UNIT}

- The EDR vendor did not identify and establish customer parameters for standard configuration and thresholds for the standard EDR unit operation. The result was reports with variations in data, flagging parameters exceeding established thresholds between individual buses.

- Also, due to lack of a standard configuration, the purchaser required the EDR component manufacturer to provide all bus manufacturers currently under contract with the best installation fit and system operational configurations by developing an "as built" protocol specification. FDOT had not previously experienced similar installation issues on other components in buses built by other vendors.

\section{LACK OF TRAINING}

- Training is major problem that exists from the component manufacturer down to the users because EDR systems in transit vehicles are a relatively new technology. The lack of installation and configuration training for assembly line workers at the factory was apparent. Also, the lack of training for users on how to correct errors on the unit, maintain the unit in top operational status, and collect and analyze data has resulted in a loss of interest in the EDR systems by transit agencies. The training provided to date has been minimal and more of a "sales pitch." 
FIX: The vendor and FDOT will be developing a more in-depth training program to deliver to all customers, and will provide presentations and informational items at Florida transit system conferences to increase awareness 


\section{CHAPTER 4}

\section{LESSONS LEARNED}

EDRs have been available on Florida TRIPS contracts for more than seven years. The original purpose for the placement of EDRs was to monitor the g-forces exerted on vehicles in rollover and side impact accidents to support and provide data to research supported by FDOT. Data collected from the EDR would help in accident re-creation/analysis and provide some measure of litigation protection for transit agencies. A secondary goal was that proof of installation could help reduce the cost of insurance for those properties using them. As the technology improved, enabling the capture and logging of mechanical processes such as wheelchair deployment and turn signal and brake use, the emphasis was shifted to these areas. The equipment sold today as part of the TRIPS contract enables the end-user to select up to 8 out of 12 inputs to monitor, with assurance that the data can be retrieved with little effort. Additionally, other options, with additional costs, such as operator tagging, GPS, and mapping, are available on each contract but are not part of the standard package.

The TRIPS program is taking the lead nationally to provide airline-type "black boxes" to assist in identifying crash information, analyze operator actions, and identify maintenance fleet issues. It is anticipated that the Federal Transit Administration will require EDR systems on public transit buses in the near future. Florida will be well prepared as a leader in its development and installation.

As noted from the previous chapter, the current EDR units being installed on most TRIPS contracts have been riddled with problems, and their reliability, data extraction process, and overall usefulness have been significantly less than expected. Unspecified installation procedures, lack of vendor oversight of manufacturers, failure to identify effects of changes to new chassis on the EDR system operation, hardware design, and lack of end-user training are among the leading contributors to this lack of use and popularity among public transit systems. If a transit system purchases and implements all available options, the current EDR system appears extremely technical and complex and requires a dedicated person to become the staff expert in EDR operation. 
To correct most of these problems, TRIPS is requiring the EDR vender to re-check each bus delivered since 2006. This in-field campaign includes upgrading software to the latest version, adjusting the configuration to the established TRIPS threshold standard, replacing and standardizing harness wire bundles, and adding a filter or divider to improve speed and odometer recording where necessary. Additionally, each agency is provided cabling for a laptop to download data, EDR software is installed on the transit agency laptop, and basic operating training is provided to transit system representatives. Once all buses in Florida have been certified in the above manner, more in-depth training will be developed and provided on a regional basis.

\section{RECOMMENDATIONS}

- TRIPS contracts are with local Florida bus dealers who partner with individual second stage vehicle manufacturers and modifiers. Each dealer has a Warranty and Repair Department. It is imperative that the Dealer Vehicle Technician understand the EDR system, has a laptop with EDR software, and is trained to set configuration and diagnose and repair defects.

- If EDR system becomes mandatory under federal rule and FTA will require EDR systems as standard on all public transit buses, FDOT should consider including the EDR system in Chapter 14-90, Florida Administrative Code, recognizing its importance to safety and establish standards for inspecting the system and correcting defects.

- The packaging, storage, and various uses of the data have not been fully defined and communicated effectively to the end-user, further blurring the original intent of the program and contributing to the ineffectiveness of the project. This can be improved with the development and delivery of a statewide training program. 
- It is suggested that the EDR manufacturer should develop a Preventive Maintenance procedure enabling a fleet technician to test and repair the system. This should be conducted at least once per year in conjunction with the Annual Safety Inspection, if not at more frequent PM intervals. A properly working EDR system is crucial to its data being accepted as evidence in a legal action.

- TRIPS staff have been actively researching and considering the use of other EDR manufacturer systems and have included a different vendor system with g-force, sensor option, and camera/audio recording in one of its contracts. This company has shown it is capable of working closely with second stage manufacturers.

- For any EDR unit to work properly and for the user to have confidence in the data, regardless of EDR vendor, sensors, cameras or a combination of the two, all require pre-wiring during manufacturing of the passenger compartment in the $\mathrm{cab}$ and chassis. It is imperative that the component vendor work closely with the OEM and the second stage manufacturer to ensure that an installation protocol is developed satisfactory to the vehicle manufacturers and component vendor. The second stage manufacturer needs to be familiar with the installation, wiring, operation, and configuration of the EDR unit. The pre-wiring and the EDR unit must be wired correctly at the factory or it will not matter what EDR manufacturer is used, there will be problems upon delivery to customers.

- Regardless of the EDR manufacturer selected, the EDR units must be tested for a period of time to ensure proper installation was conducted at the factory. A number of agencies should be selected as testing sites for the EDR units to ensure that the units are working properly with different chassis and the EDR units are meeting the expectations of program managers and users before full fleet deployment of the EDR system. 
- The EDR system, at a minimum, must record only those inputs that would be used for rollover or side impact, as part of significant accident investigation. Sensors to be monitored should include:
o Date
o Time of Day
$\mathrm{O} \mathrm{MPH}$
o Acceleration
o Brake
O G-force-Accelerometers (3).

- EDR should continue to be included as a component of a base bus. The end-user should be informed that it is on the bus, but an option should be provided to the agency to notify FDOT to assist in the analysis of EDR system accident data. FDOT would ensure that the EDRs worked as promised and would monitor the project with periodic data extraction and evaluation. FDOT would extract data in the event of an accident if a system requested assistance.

- Another option would be to install the EDR base system and offer the end-user the ability to monitor several inputs. The operational aspects of the project would be the responsibility of the end-user, who would then coordinate with the EDR manufacturer for required support. Because of the uniqueness of the product and software, other entities could get involved when data are sought for analysis (end-user IT department), which makes "one size fits all" remedies difficult at best.

- The current process should be continued, but the inputs to monitor should be designated (same on all buses) and support should be provided to ensure the system works as advertised. An EDR representative should be required to be on site when the system is installed on the initial bus orders at the factory and should ensure the system functions properly when it leaves Tallahassee. The 
vendor should be required to annually audit each manufacturer for adherence to the "As-Built" protocol.

- EDR vendor products may provide detailed data and information for analysis, but the systems are not very user friendly. Most EDR systems would require a dedicated staff person if continuous data review was desired. Other transit software applications provide exception reporting based on exceeding thresholds established by the user. This would enable a daily "Exception Report" to be provided to the accident investigator, the fleet manager, the operations manager, and the operator trainer, for example. Each staff person could decide if more detailed data and further investigation would be needed for any bus identified with a threshold exception. This is only one example of the steps EDR vendors should take to make their systems more simplistic and less overwhelming to the daily user. 


\section{REFERENCES}

A Review of Jurisprudence Regarding Event Data Recorders: Implications for the Access and Use of Data for Transport Canada Collision Investigation, Reconstruction, Road Safety Research, and Regulation, prepared for the Road Safety and Motor Vehicle Regulation, Transport Canada. March 31, 2005

Development of Requirements and Functional Specifications for Crash Event Data Recorders Final Report, John Pierowicz, Daniel P. Fuglewicz, Glenn Wilson, General Dynamics, Advanced Information Systems. December 2004.

History of EDR's, Current Uses, Issues, Current and Future Mandates, FDOT/FPTA/CUTR Professional Development Workshop, Geoff Lawrence, Truck, Trailer and Transit, June 2006.

The Double Edged Sword that is the Event Data Recorder, Andrew Askland, College of Law, Arizona State University, 2006.

Transportation Recorders on Commercial Vehicles, Paul Menig and Cary Coverdill, Freightliner Corporation, 1998.

U.S. Dept. of Transportation, National Highway Traffic Safety Administration, Final Rule, 49 CFR Part 563, Event Data Recorders, Aug. 21, 2006.

Use of Event Data Recorder (EDR) Technology for Highway Crash Data Analysis, Transportation Research Board NCHRP (Project 17-24), Transportation Research Board, December 2004.

Vehicle Data Recorders - FMCSA-PSV-06-001, Federal Motor Carrier Safety Administration, December 2005. 